\title{
REPRESENTATIONS OF SYMMETRIC LINEAR DYNAMICAL SYSTEMS*
}

\author{
FABIO FAGNANI ${ }^{\dagger}$ AND JAN WILLEMS ${ }^{\ddagger}$
}

\begin{abstract}
The purpose of this paper is to study static symmetries in linear time-invariant differential dynamical systems. The main result is a representation theorem which brings the symmetry strongly into evidence. This result is then applied to a number of examples involving permutations and rotations. We close by proving a general result on the representation of compact groups on the ring of unimodular polynomial matrices.
\end{abstract}

Key words. linear systems, symmetry, representations, canonical forms, group representations, permutations, rotations

AMS subject classifications. 93A, 93B

1. Introduction. Symmetry is a very appealing concept in many scientific endeavors. It plays a major role particularly in physics and in chemistry (for example, in crystallography). It has also been extensively studied in the classical theory of dynamical systems. A salient result in this area is Noether's theorem showing the equivalence of symmetries and conservation laws in Hamiltonian dynamics.

Also, many control problems will exhibit symmetry. For example, it is of interest to ascertain if a platform suspended on four pivots with a $90^{\circ}$ rotation symmetry can be adequately stabilized by a control mechanism that also has this symmetry. Many mechanical systems will have a rotation symmetry, and an analogous question occurs in this case. A classical control problem that can be viewed as a symmetry question is whether an optimal controller for a time-invariant system will itself be time-invariant.

Although some interesting work has been done on symmetry questions in control, it is not a standard problem area. Notable contributions are the papers by Hazewinkel and Martin [5], [6] and Martin [8] motivated by certain questions related to the stabilization of linear systems by means of symmetric feedback control laws. Other places in control where symmetry problems have been studied are [1], [4], [11]-[13]. These authors are mainly concerned with nonlinear systems.

The purpose of the present paper is a fundamental study of symmetry in the context of linear systems described by differential equations. We will mainly consider representation questions. In a later paper, we plan to apply these results to control problems. The mathematical formulation follows the setting proposed in [14]. In a sense, the paper is a sequel to [2], where an elegant representation result has been obtained for time-reversible systems (cf. Theorem 2). In the present paper, we will study static symmetries and apply the representation results obtained especially to systems that are invariant under permutations or under rotations.

In an essential way, the paper uses the theory of group representations, a rather abstract area of mathematics whose original motivation lies very much in various aspects of symmetry. For an introduction to the theory of group representations, refer to [9].

* Received by the editors June 10, 1991; accepted for publication (in revised form) April 29, 1992. This research was supported by a collaborative grant from the Netherlands Organization for the Advancement of Scientific Research (NWO) and the Consiglio Nazionale dell Ricerche (CNR) and the European Economic Community Science Program contract SCI-0433-C(A).

$\dagger$ Scuola Normale Superiore, Piazza dei Cavalieri, 56100 Pisa, Italy.

¥ Mathematics Institute, University of Groningen, P.O. Box 800, 9700 AV Groningen, the Netherlands. 
We close this introduction with a few words about notation and nomenclature.

Throughout, $\mathbb{K}$ will denote $\mathbb{R}$ or $\mathbb{C}$. Some of our results will be rather different for $\mathbb{R}$ and for $\mathbb{C}$. $\mathbb{K}^{n}$ denotes the $n$-dimensional (column) vectors over $\mathbb{K}$, and $\mathbb{K}^{n_{1} \times n_{2}}$ denotes the matrices over $\mathbb{K}$ with $n_{1}$ rows and $n_{2}$ columns. We will always consider vectors as columns and occasionally write the $n$-column vector $x$ in term of its components as $\operatorname{col}\left(x_{1}, x_{2}, \ldots, x_{n}\right)$. A composite matrix will be written as $M=\left[M_{1} \vdots M_{2}\right]$, and so forth; $\operatorname{diag}\left(m_{1}, m_{2}, \ldots, m_{n}\right)$ denotes then $n \times n$ diagonal matrix with $(i, i)$ th element $m_{i}$. A similar notation will be used for block diagonal matrices. The determinant of a matrix is denoted as det.

Let $f: A \rightarrow B$. For $A^{\prime} \subset A$, the restriction of $f$ to $A^{\prime}$ will be denoted as $\left.f\right|_{A^{\prime}}$. The map that identifies an element of $A^{\prime} \subset A$ with the same element in $A$ will be called the canonical injection. ker means kernel, and im means image. The set of infinitely differentiable maps from $A$ to $B$ will be denoted as $C^{\infty}(A ; B)$.

The set of polynomial matrices over $\mathbb{K}$ with $n_{1}$ rows and $n_{2}$ columns in the indeterminate $s$ will be denoted by $\mathbb{K}^{n_{1} \times n_{2}}[s] ; \mathbb{K}^{\bullet \times n}[s]$ denotes the set of polynomial matrices with $n$ columns and any (of course, finite) number of rows. An element $R \in \mathbb{K}^{n_{1} \times n_{2}}[s]$ is said to be of full row rank if it contains a $n_{1} \times n_{1}$ submatrix with determinant nonzero. We will denote the set of full row rank elements of $\mathbb{K}^{\bullet \times n}[s]$ by $\mathbb{K}_{f r}^{\bullet \times n}[s] ;$ of $\mathbb{K}_{f r}^{n_{1} \times n_{2}}[z s]$ denotes the elements of $\mathbb{K}_{f r}^{\bullet \times n_{2}}[s]$ with $n_{1}$ rows.

Let $\mathcal{R}$ be a ring with an identity. An element $U \in \mathcal{R}$ is said to be unimodular if there exists $U^{-1} \in \mathcal{R}$ such that $U U^{-1}=U^{-1} U$ is equal to the identity. The unimodular elements of $\mathcal{R}$ clearly form a multiplicative group, called the group of units of $\mathcal{R}$. The set of $n \times n$ matrices over $\mathcal{R}$ also forms a ring. Its group of units will be denoted by $G L(n, \mathcal{R})$. The following two examples will be very important to us throughout the paper.

1. $G L(n, \mathbb{K})$, the set of nonsingular elements of $\mathbb{K}^{n \times n}$;

2. $G L(n, \mathbb{K}[s])$, the set of unimodular $(n \times n)$ polynomial matrices. Thus $U \in$ $\mathbb{K}^{n \times n}[s]$ belongs to $G L(n, \mathbb{K}[s])$ if and only if its determinant is nonzero and belongs to $\mathbb{K}$, i.e., if it is a nonzero constant.

The set of isomorphisms on the vector space $V$ is denoted by $G L(V)$. Thus, by considering the matrix representation of elements of $G L\left(\mathbb{K}^{n}\right)$ with respect to the standard basis, $G L\left(\mathbb{K}^{n}\right) \cong G L(n, \mathbb{K})$. As such, we will not make a distinction between these two sets and use $G L\left(\mathbb{K}^{n}\right)$ even where it may be more natural to write $G L(n, \mathbb{K})$.

Let $M$ be a set. A parametrization $(P, \pi)$ of $M$ consists of a set $P$ and a surjective map $\pi: P \rightarrow M$. The set $P$ is called the parameter space. Typically, $M$ is an abstract set, while $P$ consists of concrete objects (as matrices or polynomial matrices -in which case, we refer to a matrix parametrization or a polynomial matrix parametrization of $M)$. Note that $\pi$ is surjective but not necessarily bijective. If $\pi$ is a bijection, we will call the parametrization trim. In any case, the map $\pi: P \rightarrow M$ leads to the equivalence relation $E$ on $P$ defined by $\left(p_{1} E p_{2}\right): \Leftrightarrow\left(\pi\left(p_{1}\right)=\pi\left(p_{2}\right)\right)$. This equivalence relation leads to canonical forms and to invariants. A subset $P_{c} \subseteq P$ will be called a canonical form for the parametrization $(P, \pi)$ if $\pi\left(P_{c}\right)=M$, i.e., if $\left(P_{c}, \pi \mid P_{c}\right)$ is also parametrization of $M$. It is a trim canonical form if $\left.\pi\right|_{P_{c}}: P_{c} \rightarrow M$ is a bijection.

2. Differential dynamical systems. Following the terminology explained in [15], we will define a dynamical system $\Sigma$ to consist of a triple, $\Sigma=(\mathbb{T}, \mathbb{W}, \mathcal{B})$, with $\mathbb{T}$ a subset of $\mathbb{R}$, called the time axis; $\mathbb{W}$ a set called the signal space; and $\mathcal{B}$ a subset of $\mathbb{W}^{\mathbb{T}}(:=$ all maps from $\mathbb{T}$ to $\mathbb{W})$, called the behavior. Thus the behavior consists of a given family of trajectories $w: \mathbb{T} \rightarrow \mathbb{R}$.

We will consider continuous-time dynamical systems with time axis $\mathbb{T}=\mathbb{R}$ and 
with signal space $\mathbb{W}=\mathbb{K}^{q}$, with $\mathbb{K}=\mathbb{R}$ (the real case) or $\mathbb{K}=\mathbb{C}$ (the complex case). We will treat both cases in parallel. As we will see, there are distinct advantages, stemming from the theory of group representations, not to limit attention to the real case, although, admittedly, it is the real case that is of interest in applications. However, in $\S 7.4$ we see that rotation symmetries actually lead to complex systems!

The dynamical system $\Sigma=\left(\mathbb{R}, \mathbb{K}^{q}, \mathcal{B}\right)$ is said to be linear if $\mathcal{B}$ is a linear subspace of $\left(\mathbb{K}^{q}\right)^{\mathbb{R}}$ (the set of all maps from $\mathbb{R}$ to $\mathbb{K}^{q}$ ) and time-invariant if $\sigma^{t} \mathcal{B}=\mathcal{B}$ for all $t \in \mathbb{R} ; \sigma^{t}$ denotes the backward $t$-shift (specifically, for $f: \mathbb{R} \rightarrow \mathbb{K}^{q}$ and $t \in \mathbb{R}$, $\sigma^{t} f: \mathbb{R} \rightarrow \mathbb{K}^{q}$ is defined by $\left.\left(\sigma^{t} f\right)\left(t^{\prime}\right):=f\left(t+t^{\prime}\right)\right)$.

In the present paper, we study behaviors $\mathcal{B}$ that are the solution set of a system of constant coefficient linear differential equations

$$
R\left(\frac{d}{d t}\right) w=0
$$

defined in terms of a polynomial matrix $R \in \mathbb{K}^{\bullet \times q}[s]$. The solution set of (1) is formally defined as follows:

$$
\mathcal{B}=\left\{w \in C^{\infty}\left(\mathbb{R} ; \mathbb{K}^{q}\right) \mid\left(R\left(\frac{d}{d t}\right) w\right)(t)=0 \text { for all } t \in \mathbb{R}\right\}
$$

The assumption that $w$ is infinitely differentiable is used mainly for convenience. The results may be generalized without difficulty to the case that $\mathcal{B}$ also allows locally integrable functions, or distributions. However, for the purposes of the present paper, the smoothness assumption simplifies the analysis somewhat. In other applications, the $C^{\infty}$ assumption may be very awkward.

The class of dynamical systems studied in this paper consists of those whose behavior is the kernel of a constant coefficient linear differential operator (with, for $R \in \mathbb{K}^{p \times q}[s], R(d / d t)$ viewed as a map from $C^{\infty}\left(\mathbb{R} ; \mathbb{K}^{q}\right)$ to $\left.C^{\infty}\left(\mathbb{R} ; \mathbb{K}^{p}\right)\right)$. We will denote this class of dynamical systems as $\mathcal{L}^{q}$ and refer to its elements as differential dynamical systems.

The above shows that $\left(\mathbb{K}^{\bullet \times q}[s], \pi\right)$ is a parametrization of $\mathcal{L}^{q}$ with for $R \in$ $\mathbb{K}^{\bullet \times q}[s], \pi(R):=\left(\mathbb{R}, \mathbb{K}^{q}\right.$, ker $\left.R(d / d t)\right)$. This induces the equivalence relation $\sim$ on $R^{\bullet \times q}[s]$ defined by $\left(R_{1} \sim R_{2}\right): \Leftrightarrow\left(\pi\left(R_{1}\right)=\pi\left(R_{2}\right)\right)$. Note, in fact, that $\pi$ is not injective. Indeed, if $R \in \mathbb{K}^{p \times q}[s]$ and $U \in G L(p, \mathbb{K}[s])$ (thus $U$ is unimodular), then clearly $U R \sim R$.

We will call the system of differential equations (1) or, equivalently, $R$, a behavioral equation representation of $\pi(R) ;(1)$ or, equivalently, $R$ is called a minimal (behavioral equation) representation of $\pi(R)$ if $\left(R_{1} \in \mathbb{K}^{p_{1} \times q}[s], R \in \mathbb{K}^{p \times q}[s]\right.$, and $R_{1} \sim R$ ) implies $\left(p_{1} \geq p\right)$. Let $\Sigma \in \mathcal{L}^{q}$ and let $R$ be a minimal behavioral equation of $\Sigma$. Obviously, the number of rows of $R \in \mathbb{K}^{\bullet \times q}[s]$ will depend only on $\Sigma$ but not on the particular minimal representation $R$ of $\Sigma$. We will denote the number of rows of $R$ by $p(\Sigma)$. Actually, $p(\Sigma)$ is equal to the number of output variables in any input/output representation of $\Sigma$ (see [10]).

The following characterization of minimal representations will play an important role throughout the paper.

Proposition 1. (1) is minimal if and only if $R \in \mathbb{K}_{f r}^{\bullet \times q}[s]$ (that is, $R \in \mathbb{K}^{\bullet \times q}[s]$ is of full row rank). Moreover, if (1) is minimal and if $R_{1} \in \mathbb{K}^{\bullet \times q}[s]$, then $\left(R_{1} \sim\right.$ $R$ and $R_{1}$ is also minimal $) \Leftrightarrow\left(R_{1}\right.$ and $R$ both belong to $\mathbb{K}^{p(\Sigma) \times q}[s]$, and there exists a $U \in G L(p(\Sigma), \mathbb{K}[s])$ such that $\left.R_{1}=U R\right)$. Finally, this $U$ is unique.

Proof. For the proof, see [10]. 
This proposition implies that all minimal representations may be obtained from one by acting (as premultiplication) with the unimodular group. The freedom that this implies on the representations of a given dynamical system in terms of (minimal) behavioral equations will allow us to choose $R$ 's in (1) that have an appealing form, reflecting symmetries.

3. Symmetric systems. The purpose of this paper is to study symmetries of dynamical systems in $\mathcal{L}^{q}$. A symmetry is induced by a transformation group, the basic idea being that we have a group of transformations mapping a dynamical system $\Sigma=\left(\mathbb{R}, \mathbb{K}^{q}, \mathcal{B}\right) \in \mathcal{L}^{q}$ into another such dynamical system. If this transformation does not change $\Sigma$, then we will call $\Sigma$ symmetric. We will now formalize this.

3.1. Transformation groups. Let $S$ be a set and $G$ be a group. Let $T$ be a map from $G$ into the group of bijections on $S$. We will denote the $T$-image of $g \in G$ by $T_{g}$. Then $T$ is said to be a transformation group on $S$ if $T$ is a group homomorphism, that is, if $T_{g_{1} g_{2}}=T_{g_{1}} T_{g_{2}}$. (The multiplication $g_{1} g_{2}$ refers to the multiplication in the group $G$, while $T_{g_{1}} T_{g_{2}}$ refers to composition of maps on $S$.) For $s \in S$, the set $O_{s}:=\left\{s^{\prime} \in S \mid \exists g \in G\right.$ such that $\left.s^{\prime}=T_{g} s\right\}$ is called the orbit through $s$. It is easily seen that, for $s_{1}, s_{2} \in S$, either $O_{s_{1}}=O_{s_{2}}$, or $O_{s_{1}} \cap O_{s_{2}}=\emptyset$, the first situation occuring if and only if $s_{2} \in O_{s_{1}}$. The collection of orbits $\left\{O_{s} \mid s \in S\right\}$ hence defines a partition of $S$ and thus an equivalence relation on $S$.

3.2. Symmetries. Let $T$ be a transformation group on $\mathcal{L}^{q}$. We will call the dynamical system $\Sigma \in \mathcal{L}^{q}, T$-symmetric if $T_{g} \Sigma=\Sigma$ for all $g \in G$. Thus, for a symmetric element $\Sigma$, the orbit $O_{\Sigma}$ is equal to the singleton $\{\Sigma\}$.

Let us now consider a few examples of symmetries on $\mathcal{L}^{q}$.

Example 1 (time-invariance). Let $G=\mathbb{R}$ and define, for $\Sigma=\left(\mathbb{R}, \mathbb{K}^{q}, \mathcal{B}\right) \in \mathcal{L}^{q}, T_{g} \Sigma$ as $T_{g} \Sigma=\left(\mathbb{R}, \mathbb{K}^{q}, \sigma^{g} \mathcal{B}\right.$ ) (with $\sigma^{g}$ the backward $g$-shift). It is easy to see that $T_{g} \Sigma=\Sigma$ for all $g \in \mathbb{R}$, and hence all elements of $\mathcal{L}^{q}$ are symmetric in this sense. It is this symmetry that we call time-invariance. It formalizes the fact that the laws governing a dynamical system do not depend explicitly on time.

Example 2 (time-reversibility). Many examples of symmetries involve the group consisting of only two elements, $G=\{1, g\}, 1 \neq g=g^{-1}$. Then $T_{g}=\left(T_{g}\right)^{-1}$; i.e. $T_{g}$ is an involution. Define, for $\Sigma \in \mathcal{L}^{q}, T_{g} \Sigma$ as $T_{g} \Sigma:=\left(\mathbb{R}, \mathbb{R}^{q}, \operatorname{rev} \mathcal{B}\right)$ with for $w: \mathbb{R} \rightarrow \mathbb{R}^{q}$, $\operatorname{rev} w: \mathbb{R} \rightarrow \mathbb{R}^{q}$, the time-reverse of defined by $(\operatorname{rev} w)(t):=w(-t)$. This $\Sigma$ will be symmetric with respect to this transformation group if and only if $\mathcal{B}=\operatorname{rev} \mathcal{B}$. This symmetry is called time-reversibility. It expresses the fact that the system looks identical when viewed backward in time. We have studied this symmetry in detail in [2] and will return to it later in this paper.

3.3. Static symmetries. Let $T$ be a transformation group acting on $\mathbb{K}^{q} ; T$ induces a symmetry on $\mathcal{L}^{q}$ by defining for $\Sigma=\left(\mathbb{R}, \mathbb{K}^{q}, \mathcal{B}\right) \in \mathcal{L}^{q}, T_{g} \Sigma$ as $T_{g} \Sigma:=$ $\left(\mathbb{R}, \mathbb{K}^{q}, T_{g} \mathcal{B}\right)$ with $T_{g} \mathcal{B}:=\left\{w: \mathbb{R} \rightarrow \mathbb{R}^{q} \mid \exists w^{\prime}: \mathbb{R} \rightarrow \mathbb{K}^{q}\right.$ such that $w(t)=T_{g} w^{\prime}(t)$ for all $t \in \mathbb{R}\}$. Note that, by a minor abuse of notation, we use the same symbol $T_{g}$ as acting on $\mathcal{L}^{q}$, on $\mathcal{B}$, and on $\mathbb{K}^{q}$. Thus $\Sigma$ is symmetric in this sense if $w \in \mathcal{B}$ implies $T_{g} w \in \mathcal{B}$ for all $g \in G$. Since $T_{g}$ transforms the trajectories $w$ in $\mathcal{B}$ by applying the memoryless map $T_{g}$ (that is, since it transforms trajectories $w$ in a nondynamic way), we will call such a symmetry a static symmetry. In fact, we will be particularly interested in the case where $T_{g}$ is linear for all $g \in G$. Such transformation groups are the subject of the theory of group representations. It is customary to denote $T$ by $\rho$ in that case. 
3.4. Group representations. Let $V$ be a vector space over the field $\mathbb{K}$. A group homomorphism $\rho: G \rightarrow G L(V)$ is said to be a (linear) representation of the group $G$ on $V$. If $V$ is finite-dimensional, then the representation is called finitedimensional, and the dimension of $V$ as a vector space is called the order of the representation. In particular, if $V$ is $n$-dimensional and if we represent elements of $G L(V)$ as matrices with respect to a fixed basis on $V$, then a representation of $G$ will correspond to each element of the group $G$, a nonsingular $(n \times n)$ matrix over $\mathbb{K}$ such that group multiplication goes over in multiplication of matrices. In particular, the identity matrix will correspond to the unit element in $G$.

Example 3 (permutations). As a specific example of a static symmetry, let $S_{q}$ denote the group of permutations of $q$ elements. This group is called the symmetric group; it is a finite group consisting of $q$ ! elements. Now consider the map $\rho$ : $S_{q} \rightarrow G L\left(\mathbb{K}^{q}\right)$, which associates with the permutation $g:\{1,2, \ldots, q\} \rightarrow\{1,2, \ldots, q\}$ the linear bijection on $\mathbb{K}^{q}$ that takes the vector col $\left(x_{1}, x_{2}, \ldots, x_{q}\right)$ into the vector $\operatorname{col}\left(x_{g(1)}, x_{g(2)}, \ldots, x_{g(q)}\right)$. Clearly, $\rho$ defines a representation of $S_{q}$ on $\mathbb{K}^{q}$. Thus $\rho$ in this case maps onto the group of $q \times q$ permutation matrices. A dynamical system $\Sigma=\left(\mathbb{R}, \mathbb{K}^{q}, \mathcal{B}\right)$ will be symmetric in the sense of the static symmetry induced by this representation of $S_{q}$, provided that $w=\operatorname{col}\left(w_{1}, w_{2}, \ldots, w_{q}\right) \in \mathcal{B}$ if and only if $w^{\prime}=\operatorname{col}\left(w_{1}^{\prime}, w_{2}^{\prime}, \ldots, w_{q}^{\prime}\right) \in \mathcal{B}$ with $\left(w_{1}^{\prime}, w_{2}^{\prime}, \ldots, w_{q}^{\prime}\right)$ any permutation of $\left(w_{1}, w_{2}, \ldots, w_{q}\right)$. We can think of this symmetry as occurring when $\Sigma$ models the dynamics of the positions of $q$ identical particles on the line: feasible motions will remain feasible motions after we interchange the positions of the particles. More meaningful symmetries as representations of $S_{q}$ (involving particles in the plane or in 3-space), or of subgroups of $S_{q}$, will be considered later.

Let $\rho: G \rightarrow G L(V)$ be a representation of $G$ on $V$, with $V$ a finite-dimensional vector space over $\mathbb{K}$. Throughout this paper, we will assume that $G$ is either finite or compact. In the compact case, $G$ is assumed to be a compact Hausdorff topological space with the group multiplication and the inverse continuous maps. A representation $\rho: G \rightarrow G L(V)$ is then always assumed to be continuous.

A subspace $V_{1} \subseteq V$ is said to be invariant if $\rho_{g} V_{1} \subseteq V_{1}$ for all $g \in G$. The representation $\rho$ is said to be irreducible if its only invariant subspaces are $V$ and $\{0\}$. When $V_{1}$ is invariant, then $\rho^{V_{1}}$, defined by $\rho^{V_{1}}: G \rightarrow G L\left(V_{1}\right)$ with $\rho_{g}^{V_{1}}:=\left.\rho_{g}\right|_{V_{1}}$, yields another finite-dimensional representation of $G: \rho_{1}^{V}$ is called a subrepresentation. It is a standard result form the theory of group representations that, if $G$ is compact, then a finite-dimensional subrepresentation can be written as the direct sum of irreducible representations.

Let $\rho^{1}: G \rightarrow G L\left(V_{1}\right)$ and $\rho^{2}: G \rightarrow G L\left(V_{2}\right)$ be two finite-dimensional representations of the same group $G$. Then they are said to be isomorphic if $V_{1}$ and $V_{2}$ have the same dimension and if there exists an isomorphism $S: V_{1} \rightarrow V_{2}$ such that $\rho_{g}^{2}=S \rho_{g}^{1} S^{-1}$ for all $g \in G$. Isomorphism of $\rho_{1}$ and $\rho_{2}$ will be denoted by $\rho_{1} \cong \rho_{2}$. If $\rho_{1}$ is not isomorphic to $\rho_{2}$, then $\rho_{1}$ and $\rho_{2}$ are said to be distinct.

Thus the above implies that a representation $\rho$ admits a decomposition of the following type:

$$
\rho \cong m_{1} \rho_{1} \oplus m_{2} \rho_{2} \oplus \cdots \oplus m_{k} \rho_{k}
$$

where $\rho_{1} \cdots \rho_{k}$ are distinct irreducible representation and where

$$
m_{j} \rho_{j}:=\underbrace{\rho_{j} \oplus \rho_{j} \oplus \cdots \oplus \rho_{j}}_{m_{j} \text {-times }} .
$$


Example 3 (continued). Recall that $S_{q}$ denotes the group of permutations of $q$ elements. It is a finite group containing $q$ ! elements. The irreducible representations of $S_{q}$ have been studied in much detail in the literature. However, we will need only two of them. Consider the following representations of $S_{q}$ :

1. The identity representation, $\rho_{1}: S_{q} \rightarrow G L(\mathbb{K})$ with $\rho_{1, g}=1$ for all $g \in S_{q}$. This representation is of order 1 and hence irreducible;

2. The representation $\rho_{2}$ defined as follows. Let $V$ be the subspace of $\mathbb{K}^{q}$ consisting of those vectors $\operatorname{col}\left(x_{1}, x_{2}, \ldots, x_{q}\right)$ such that $\sum_{i=1}^{q} x_{i}=0$. Let $S_{q}$ act on $V$ by $\rho_{2, g} \operatorname{col}\left(x_{1}, x_{2}, \ldots, x_{q}\right):=\operatorname{col}\left(x_{g(1)}, x_{g(2)}, \ldots, x_{g(q)}\right)$. It is easy to prove that $\rho_{2}$ defines an irreducible representation of $S_{q}$. Since $\operatorname{dim} V=q-1$, its order is $q-1$.

Let $\rho: S_{q} \rightarrow G \ell\left(\mathbb{K}^{q}\right)$ be the representation of $S_{q}$ introduced in Example 3: $\rho_{g} \operatorname{col}\left(x_{1}, x_{2}, \ldots, x_{q}\right):=\operatorname{col}\left(x_{g(1)}, x_{g(2)}, \ldots, x_{g(q)}\right)$. Write $\mathbb{K}^{q}=V_{1} \oplus V_{2}$ with $V_{1}=$ $\left\{\operatorname{col}\left(x_{1}, x_{2}, \ldots, x_{q}\right) \in \mathbb{K}^{q} \mid x_{1}=x_{2}=\cdots=x_{q}\right\}$ and $V_{2}=\left\{\operatorname{col}\left(x_{1}, x_{2}, \ldots, x_{q}\right) \in \mathbb{K}^{q} \mid\right.$ $\left.x_{1}+x_{2}+\cdots+x_{q}=0\right\}$. Clearly, $V_{1}$ and $V_{2}$ are $\rho$-invariant subspaces, $\rho^{V_{1}} \cong \rho_{1}$ and $\rho^{V_{2}} \cong \rho_{2}$. Hence, in this case, the decomposition of $\rho$ in terms of the irreducible representations of $S_{q}$ becomes $\rho \cong \rho_{1} \oplus \rho_{2}$. (We hence have $m_{1}=m_{2}=1$, while all the other $m_{i}$ 's are zero. Furthermore, $n_{1}=1$ and $n_{2}=q-1$.)

4. Representation questions for symmetric systems. Assume that $\rho: G \rightarrow$ $G L\left(\mathbb{K}^{q}\right)$ is a representation of the group $G$ on $\mathbb{K}^{q}$ and assume that $\Sigma=\left(\mathbb{R}, \mathbb{K}^{q}, \mathcal{B}\right) \in \mathcal{L}^{q}$ is symmetric in the sense of the static symmetry induced by this representation. The problem studied in this paper is the following: Can this symmetry be put into evidence by an appropriate behavioral equation representation of $\Sigma$ as (1), in which the polynomial matrix $R$ is such that this static symmetry becomes evident? Otherwise stated, we want to come up with a parametrization, with a canonical form for the behavioral equations of systems with a static symmetry.

To give an example of the type of results that we seek, we repeat the main result of [2]. This result involves time-reversibility, which, it should be noted, is not a static symmetry.

THEOREM 2. $\Sigma \in \mathcal{L}^{q}$ is time-reversible if and only if it allows a minimal behavioral equation representation (1) with $R(s)=J R(-s)$ with $J$ a matrix of the type

$$
J=\left[\begin{array}{cc}
I_{1} & 0 \\
0 & -I_{2}
\end{array}\right],
$$

where $I_{1}$ and $I_{2}$ are identity matrices.

Observe that $R(s)=J R(-s)$ is equivalent to stating that (1) consists of a number of scalar differential equations, some of which contain only even-order derivatives, while the others contain only odd-order derivatives. If the equations in (1) are indeed of this form, then time-reversibility is obvious. Note, in particular, that time-reversible systems cannot always be represented by differential equations containing only evenorder derivatives. (Actually, any representation as obtained in Theorem 2 will have the dimension of $I_{1}$ and $I_{2}$ as invariants.)

While this paper is only concerned with static symmetries, it is worthwhile noting that it is possible to view also reciprocity, a much-studied property of electrical networks [17], as a (dynamic) symmetry.

5. The main result. Assume in this section that $\rho: G \rightarrow G L\left(\mathbb{K}^{q}\right)$ is a given representation of a compact group $G$ on $\mathbb{K}^{q}$. Then $\rho$ defines a static symmetry on $\mathcal{L}^{q}$ as described in $\S 3.4 ; \Sigma=\left(\mathbb{R}, \mathbb{K}^{q}, \mathcal{B}\right) \in \mathcal{L}^{q}$ is thus $\rho$-symmetric if and only if $\rho_{g} \mathcal{B}=\mathcal{B}$ for all $g \in G$. 
Let (1) be a minimal representation for such a $\rho$-symmetric $\Sigma \in \mathcal{L}^{q}$. It then follows immediately from Proposition 1 that, for each $g \in G$, there will exist a unimodular polynomial matrix $U_{g}(s)$ such that $R(s) \rho_{g}=U_{g}(s) R(s)$. Our main result tells us that $R$ can be chosen such that $U_{g}(s)$ is a constant nonsingular matrix, thus independent of $s$ !

THEOREM 3. Let $\rho: G \rightarrow G L\left(\mathbb{K}^{q}\right)$ be a representation of the compact group $G$ on $\mathbb{K}^{q} . \Sigma \in \mathcal{L}^{q}$ is $\rho$-symmetric if and only if there exists a minimal representation $R(d / d t) w=0$ of $\Sigma$ and a representation $\rho^{\prime}: G \rightarrow G L\left(\mathbb{K}^{p(\Sigma)}\right)$ of the group $G$ on $\mathbb{K}^{p(\Sigma)}$ such that

$$
R(s) \rho_{g}=\rho_{g}^{\prime} R(s)
$$

for all $g \in G$. Moreover, $\rho^{\prime}$ will be isomorphic to a subrepresentation of $\rho$.

Proof. To prove the "if" part, assume that $R(s) \rho_{g}=\rho_{g}^{\prime} R(s)$. Then, since $\rho_{g}^{\prime}$ is an invertible matrix (hence a unimodular polynomial matrix), ker $R(d / d t)=$ ker $R(d / d t) \rho_{g}$. Hence $\rho_{g} \mathcal{B}=\mathcal{B}$ for all $g \in G ; \rho$-symmetry follows. As a general feature of the type of representation results that we seek, note that also here (as in Theorem 2) the "if" is immediate: if $R(s) \rho_{g}=\rho_{g}^{\prime} R(s)$ for all $g \in G$, then $\rho$-symmetry of (1) is basically immediate. The converse however is more difficult.

The "only if" part is based on Theorems 4 and 5 and will be proved later.

To see that $\rho^{\prime}$ is isomorphic to a subrepresentation of $\rho$, pick an element $\lambda \in \mathbb{R}$ such that $R(\lambda)$ has full row rank $p(\Sigma)$. Since $R$ is minimal and hence of full row rank as a polynomial matrix, such a $\lambda \in \mathbb{K}$ exists. Now observe that $R(\lambda) \rho_{g}=\rho_{g}^{\prime} R(\lambda)$ for all $g \in G$. Let $N:=\operatorname{ker} R(\lambda)$. Obviously, $N$ is $\rho$-invariant. Hence there exists a linear subspace $M$ of $\mathbb{K}^{q}$ such that $M$ is $\rho$-invariant and $\mathbb{K}^{q}=N \oplus M$. Therefore $\left.\left.R(\lambda)\right|_{M} \rho_{g}\right|_{M}=\left.\rho_{g}^{\prime} R(\lambda)\right|_{M}$. Since $\left.R(\lambda)\right|_{M}$ is a bijection, this shows that $\rho^{\prime}$ is isomorphic to the subrepresentation $\rho^{M}$ of $\rho$.

6. Canonical forms for symmetric systems. We will now show that establishing Theorem 3 is equivalent to establishing the existence of a very nice explicit canonical forms for symmetric systems. At this point, it becomes necessary to treat the complex case $(\mathbb{K}=\mathbb{C})$ and the real case $(\mathbb{K}=\mathbb{R})$ separately.

6.1. Complex systems. The representations $\rho: G \rightarrow G L\left(\mathbb{C}^{q}\right)$ and $\rho^{\prime}: G \rightarrow$ $G L\left(\mathbb{C}^{p(\Sigma)}\right)$ obtained in $\S 5$ can be decomposed in terms of irreducible ones as

$$
\begin{gathered}
\rho \cong m_{1} \rho_{1} \oplus m_{2} \rho_{2} \oplus \cdots \oplus m_{k} \rho_{k}, \\
\rho^{\prime} \cong m_{1}^{\prime} \rho_{1} \oplus m_{2}^{\prime} \rho_{2} \oplus \cdots \oplus m_{k}^{\prime} \rho_{k} .
\end{gathered}
$$

Since $\rho^{\prime}$ is isomorphic to a subrepresentation of $\rho$, it follows that the integers $m_{i}^{\prime} \in \mathbb{Z}_{+}$ satisfy

$$
0 \leq m_{i}^{\prime} \leq m_{i}, \quad i=1,2, \ldots, k
$$

The above decomposition of $\rho$ implies that there exists a nonsingular matrix $V \in \mathbb{C}^{q \times q}$ such that

$$
V \rho_{g} V^{-1}=\operatorname{diag}\left(m_{1} \rho_{1, g}, \ldots, m_{k} \rho_{k, g}\right)=: \tilde{\rho}_{g},
$$

where

$$
m_{i} \rho_{i, g}:=\operatorname{diag}(\underbrace{\rho_{i, g}, \ldots, \rho_{i, g}}_{m_{i}-\text { times }}) .
$$


Proceeding in a similar manner for $\rho^{\prime}$, we obtain a nonsingular matrix $V^{\prime} \in$ $\mathbb{C}^{p(\Sigma) \times p(\Sigma)}$ such that

$$
V^{\prime} \rho_{g}^{\prime}\left(V^{\prime}\right)^{-1}=\operatorname{diag}\left(m_{1}^{\prime} \rho_{1, g}, m_{2}^{\prime} \rho_{2, g}, \ldots, m_{k}^{\prime} \rho_{k, g}\right)=: \tilde{\rho}_{g}^{\prime} .
$$

Note that applying the nonsingular transformation $V$ corresponds to changing the signal variables in $\Sigma=\left(\mathbb{R}, \mathbb{C}^{q}, \mathcal{B}\right)$ from $w: \mathbb{R} \rightarrow \mathbb{C}^{q}$ to $\tilde{w}: \mathbb{R} \rightarrow \mathbb{C}^{q}$ with $\tilde{w}(t):=V w(t) ;$ in other words, it corresponds to choosing a convenient basis in the signal space $\mathbb{C}^{q}$. We will call such a basis a $\rho$-adapted basis, and the corresponding coordinates $\rho$ adapted coordinates (these are sometimes called normal coordinates). On the other hand, premultiplying $R$ in (1) by nonsingular matrix $V^{\prime}$ corresponds to choosing a convenient basis in the equation space $\mathbb{C}^{p(\Sigma)}$. By Proposition 1 , this does not change the behavior, and hence we can always assume that we are using such a basis on the equation space.

Now, assume that $\rho$ and $\rho^{\prime}$ satisfy the conditions of Theorem 3. It follows thus that in a $\rho$-adapted basis the system $\Sigma=\left(\mathbb{R}, \mathbb{C}^{q}, \mathcal{B}\right) \in \mathcal{L}^{q}$ will admit a minimal representation

$$
\tilde{R}\left(\frac{d}{d t}\right) \tilde{w}=0
$$

where $\tilde{R} \in \mathbb{C}^{p(\Sigma) \times q}[s]$ satisfies

$$
\tilde{R}(s) \tilde{\rho}_{g}=\tilde{\rho}_{g}^{\prime} \tilde{R}(s) .
$$

Now partition $\tilde{R}$ conformably as $\tilde{\rho}$ and $\tilde{\rho}^{\prime}$, yielding

$$
\tilde{R}(s)=\left[\begin{array}{cccc}
\tilde{R}_{11}(s) & \tilde{R}_{12}(s) & \cdots & \tilde{R}_{1 k}(s) \\
\tilde{R}_{21}(s) & \tilde{R}_{22}(s) & \cdots & \tilde{R}_{2 k}(s) \\
\vdots & \vdots & \ddots & \vdots \\
\tilde{R}_{k 1}(s) & \tilde{R}_{k 2}(s) & \cdots & \tilde{R}_{k k}(s)
\end{array}\right]
$$

Then (3) implies that $\tilde{R}_{i j}(s) m_{j} \rho_{j}=m_{i}^{\prime} \rho_{i} \tilde{R}_{i j}(s)$. By the Schur lemma [9], these equalities imply the following strong conclusions about $\tilde{R}$ :

$$
\tilde{R}_{i j}=0 \quad \text { for } i \neq j
$$

and

$$
\tilde{R}_{i i}(s)=\left[\begin{array}{cccc}
\lambda_{11}(s) I_{n_{i}} & \lambda_{12}(s) I_{n_{i}} & \cdots & \lambda_{1 m_{i}}(s) I_{n_{i}} \\
\lambda_{21}(s) I_{n_{i}} & \lambda_{22}(s) I_{n_{i}} & \cdots & \lambda_{2 m_{i}}(s) I_{n_{i}} \\
\vdots & \vdots & \ddots & \vdots \\
\lambda_{m_{i}^{\prime} 1}(s) I_{n_{i}} & \lambda_{m_{i}^{\prime} 2}(s) I_{n_{i}} & \cdots & \lambda_{m_{i}^{\prime} m_{i}}(s) I_{n_{i}}
\end{array}\right]
$$

where $n_{i}$ is the order of the representation $\rho_{i}$. In the Kronecker product notation, $\tilde{R}_{i i}$ may be written as

$$
\tilde{R}_{i i}(s)=\Lambda_{i}(s) \otimes I_{n_{i}}
$$

where

$$
\Lambda_{i}(s)=\left[\begin{array}{cccc}
\lambda_{11}(s) & \lambda_{12}(s) & \cdots & \lambda_{1 m_{i}}(s) \\
\lambda_{21}(s) & \lambda_{22}(s) & \cdots & \lambda_{2 m_{i}}(s) \\
\vdots & \vdots & \ddots & \vdots \\
\lambda_{m_{i}^{\prime} 1}(s) & \lambda_{m_{i}^{\prime} 2}(s) & \cdots & \lambda_{m_{i}^{\prime} m_{i}}(s)
\end{array}\right]
$$


This proves that, for $\mathbb{K}=\mathbb{C}$, Theorem 3 is equivalent to the following theorem.

THEOREM 4. Let $G$ be a compact group and let $\rho: G \rightarrow G L\left(\mathbb{C}^{q}\right)$ be a representation of $G$ on $\mathbb{C}^{q}$. Assume that $\rho \cong m_{1} \rho_{1} \oplus m_{2} \rho_{2} \oplus \cdots \oplus m_{k} \rho_{k}$, with $\rho_{i}$ : $G \rightarrow G L\left(\mathbb{C}^{n_{i}}\right), i=1,2, \ldots, k$, distinct irreducible representations. Assume that the basis in $\mathbb{C}^{q}$ is $\rho$-adapted (to emphazise this, we write the signal variables as $\tilde{w}$, $\tilde{w}=\operatorname{col}\left(\tilde{w}_{1}, \tilde{w}_{2}, \ldots, \tilde{w}_{k}\right)$ with $\left.\tilde{w}_{i}: \mathbb{R} \rightarrow\left(\mathbb{C}^{n_{i}}\right)^{m_{i}}\right)$. Then $\Sigma=\left(\mathbb{R}, \mathbb{C}^{q}, \mathcal{B}\right) \in \mathcal{L}^{q}$ is $\rho$ symmetric if and only if there exist $m_{i}^{\prime} \in \mathbb{Z}_{+}, 0 \leq m_{i}^{\prime} \leq m_{i}$, and polynomial matrices $\Lambda_{i} \in \mathbb{C}_{f r}^{m_{i}^{\prime} \times m_{i}}[s]$ such that $\Sigma$ admits a minimal representation

$$
\left(\Lambda_{i}\left(\frac{d}{d t}\right) \otimes I_{n_{i}}\right) \tilde{w}_{i}=0, \quad i=1,2, \ldots, k
$$

Note that, from the above theorem, we may conclude that the $\rho$-adapted variables $\tilde{w}_{1}, \tilde{w}_{2}, \ldots, \tilde{w}_{k}$ are completely noninteracting!

6.2. Real systems. In the case $(\mathbb{K}=\mathbb{R})$ of systems with signal space $\mathbb{R}^{q}$, Theorem 4 remains, of course, valid but may yield a representation (7) with complex coefficients. However, in this case, we want to obtain differential equations in a canonical form analogous to (7) but with real coefficients. The theory becomes more involved, since the irreducible representations $\rho_{i}$ introduced in $\S 6.1$ are irreducible over $\mathbb{C}$ and need not be real. In particular, Schur's lemma in the form in which it was used in $\S 6.1$ would yield complex representations. Nevertheless, quite explicit results may also be obtained now.

As it is shown in [9], a irreducible representation $\rho: G \rightarrow G L\left(\mathbb{C}^{n}\right)$ can be of real type, of complex type, or of quaternionic type; $\rho$ is of real type if it is the complexification of a real representation. Now, if $\rho$ is an irreducible representation of complex or of quaternionic type, then so will be its complex conjugate, $\rho^{*}$ (thus the matrices $\rho_{g}$ and $\rho_{g}^{*}$ are complex conjugates for all $g \in G$ ). By combining $\rho \oplus \rho^{*}$, these complex representations lead to real ones. All together, this leads to the following decomposition of a real representation. sition

A real representation $\rho: G \rightarrow G \ell\left(\mathbb{R}^{q}\right)$ of a compact group $G$ admits a decompo-

$$
\rho \cong \rho_{\mathbb{R}} \oplus \rho_{\mathbb{C}} \oplus \rho_{\mathbb{H}}
$$

with $\rho_{\mathbb{R}}, \rho_{\mathbb{C}}$, and $\rho_{\mathbb{H}}$ referring to the further decomposition into irreducible representations of real, complex, or quaternionic type. These representations can indeed be further decomposed as follows:

$$
\begin{aligned}
& \rho_{\mathbb{R}}=m_{\mathbb{R}, 1} \rho_{\mathbb{R}, 1} \oplus m_{\mathbb{R}, 2} \rho_{\mathbb{R}, 2} \oplus \cdots \oplus m_{\mathbb{R}, k_{\mathbb{R}}} \rho_{\mathbb{R}, k_{\mathbb{R}}}, \\
& \rho_{\mathbb{C}}=m_{\mathbb{C}, 1} \rho_{\mathbb{C}, 1} \oplus m_{\mathbb{C}, 2} \rho_{\mathbb{C}, 2} \oplus \cdots \oplus m_{\mathbb{C}, k_{\mathbb{C}}} \rho_{\mathbb{C}, k_{\mathbb{C}}} \\
& \rho_{\mathbb{H}}=m_{\mathbb{H}, 1} \rho_{\mathbb{H}, 1} \oplus m_{\mathbb{H}, 1} \rho_{\mathbb{H}, 2} \oplus \cdots \oplus m_{\mathbb{H}, k_{\mathbb{H}}} \rho_{\mathbb{H}, k_{\mathbb{H}}}
\end{aligned}
$$

with $\rho_{\mathbb{R}, i}: G \rightarrow G L\left(\mathbb{R}^{n_{\mathbb{R}, i}}\right)$ such that its complexification is irreducible over $\mathbb{C} ; \rho_{\mathbb{C}, i}$ : $G \rightarrow G L\left(\mathbb{R}^{2 n_{\mathbb{C}, i}}\right)$ such that the complexification of $\rho_{\mathbb{C}, i}$ is isomorphic (over $\mathbb{C}$ ) to $\tilde{\rho}_{\mathbb{C}, i} \oplus \tilde{\rho}_{\mathbb{C}, i}^{*}$ with $\tilde{\rho}_{\mathbb{C}, i}: G \rightarrow G L\left(\mathbb{C}^{n_{\mathbb{C}, i}}\right)$ irreducible over $\mathbb{C} ;$ similarly, $\rho_{\mathbb{H}, i}: G \rightarrow$ $G L\left(\mathbb{R}^{4 n_{\mathbb{H}, i}}\right), \rho_{\mathbb{H}, i} \cong \tilde{\rho}_{\mathbb{H}, i} \oplus \tilde{\rho}_{\mathbb{H}, i}^{*}$ with $\tilde{\rho}_{\mathbb{H}, i}: G \rightarrow G L\left(\mathbb{C}^{2 n_{\mathbb{H}, i}}\right)$ also irreducible over $\mathbb{C}$. 
The representations $\rho_{\mathbb{R}, 1}, \ldots, \rho_{\mathbb{R}, k_{\mathbb{R}}} ; \tilde{\rho}_{\mathbb{C}, 1}, \ldots, \tilde{\rho}_{\mathbb{C}, k_{\mathbb{c}}} ; \tilde{\rho}_{\mathbb{C}, 1}^{*}, \ldots, \tilde{\rho}_{\mathbb{C}, k_{\mathbb{C}}}^{*} ; \tilde{\rho}_{\mathbb{H}, 1}, \ldots, \tilde{\rho}_{\mathbb{H}, k \mathbb{H}}$ are the distinct irreducible representations of real, complex, and quaternionic type, respectively, involved in the decomposition of $\rho$,

$n_{\mathbb{R}, 1}, \ldots, n_{\mathbb{R}, k_{\mathbb{R}}} ; n_{\mathbb{C}, 1}, \ldots, n_{\mathbb{C}, k_{\mathbb{C}}} ; n_{\mathbb{H}, 1}, \ldots, n_{\mathbb{H}, k_{\mathbb{H}}}$,

their respective orders, and

$$
m_{\mathbb{R}, 1}, \ldots, m_{\mathbb{R}, k_{\mathbb{R}}} ; m_{\mathbb{C}, 1}, \ldots, m_{\mathbb{C}, k_{\mathfrak{c}}} ; m_{\mathbb{H}, 1}, \ldots, m_{\mathbb{H}, k_{\mathbb{H}}},
$$

their respective multiplicities.

A real subrepresentation $\rho^{\prime}$ of $\rho$ will allow a similar representation as (8), but with the analogous multiplicities $m_{\mathbb{R}, i}^{\prime}, m_{\mathbb{C}, i}^{\prime}$ and $m_{\mathbb{H}, i}^{\prime}$ satisfying $0 \leq m_{\mathbb{R}, i}^{\prime} \leq m_{\mathbb{R}, i}, 0 \leq$ $m_{\mathbb{C}, i}^{\prime} \leq m_{\mathbb{C}, i}$ and $0 \leq m_{\mathbb{H}, i}^{\prime} \leq m_{\mathbb{H}, i}$.

Now, assume that $\rho$ and $\rho^{\prime}$ satisfy the conditions of Theorem 3. Proceeding as in the complex case, we obtain (in the analogous partition of $\tilde{R}$ ) that, in a basis compatible with (8), $\Sigma=\left(\mathbb{R}, \mathbb{R}^{q}, \mathcal{B}\right) \in \mathcal{L}^{q}$ will admit a (real) minimal representation as (2) with (5) still satisfied. Hence the off-diagonal blocks of $\tilde{R}$ will still be zero: the components of $\tilde{w}$ will again be noninteracting.

However, Schur's lemma allows us to conclude the simple form (6) for the diagonal blocks of $\tilde{R}$ only for the diagonal blocks corresponding to the real representations, the $\rho_{\mathbb{R}, i}$ 's. The diagonal blocks of $\tilde{R}$ corresponding to the $\rho_{\mathbb{C}, i}$ 's and the $\rho_{\mathbb{H}, i}$ 's will be more complicated, and it is here that the difference between the real, complex, and quaternionic type plays a role. To obtain a convenient form for the corresponding diagonal blocks of $\tilde{R}$, we should choose the basis in the $\mathbb{R}^{2 n_{\mathrm{C}, i}}$ 's such that $\rho_{\mathbb{C}, i}$ takes the form

$$
\rho_{\mathbb{C}, i} \cong\left[\begin{array}{rr}
A_{\mathbb{C}, i} & -B_{\mathbb{C}, i} \\
B_{\mathbb{C}, i} & A_{\mathbb{C}, i}
\end{array}\right],
$$

and in the $\mathbb{R}^{4 n_{\mathrm{H}, i}}$ 's such that $\rho_{\mathbb{H}, i}$ takes the form

$$
\rho_{\mathbb{H}, i} \cong\left[\begin{array}{rrrr}
A_{\mathbb{H}, i} & -B_{\mathbb{H}, i} & -C_{\mathbb{H}, i} & -D_{\mathbb{H}, i} \\
B_{\mathbb{H}, i} & A_{\mathbb{H}, i} & D_{\mathbb{H}, i} & -C_{\mathbb{H}, i} \\
C_{\mathbb{H}, i} & -D_{\mathbb{H}, i} & A_{\mathbb{H}, i} & B_{\mathbb{H}, i} \\
D_{\mathbb{H}, i} & C_{\mathbb{H}, i} & -B_{\mathbb{H}, i} & A_{\mathbb{H}, i}
\end{array}\right] .
$$

It can be shown that there exists a (real) choice of the basis in $\mathbb{R}^{q}$ that is compatible with the decomposition (8a) and in which the $\rho_{\mathbb{C}, i}$ 's and the $\rho_{\mathbb{H}, i}$ 's have the above form. We will call such a basis choice real $\rho$-adapted.

Schur's lemma then allows to conclude that in a real $\rho$-adapted basis in $\mathbb{R}^{q}$ and in a real $\rho^{\prime}$-adapted basis in $\mathbb{R}^{p(\Sigma)}$ we will obtain a representation (2) with

$$
\tilde{R}_{i j}(s)=0 \quad \text { for } i \neq j
$$

and

$$
\tilde{R}_{i i}(s)=\tilde{A}_{i}(s) \otimes I_{n_{\mathrm{R}, i}}
$$

for the diagonal blocks corresponding to the $\rho_{\mathbb{R}, i}$ 's

$$
\tilde{R}_{i i}(s)=\left[\begin{array}{cc}
\tilde{A}_{i}(s) & -\tilde{B}_{i}(s) \\
\tilde{B}_{i}(s) & \tilde{A}_{i}(s)
\end{array}\right] \otimes I_{n_{\mathrm{C}, i}}
$$


for the diagonal blocks corresponding to the $\rho_{\mathbb{C}, i}$ 's, and

$$
\tilde{R}_{i i}(s)=\left[\begin{array}{rrrr}
\tilde{A}_{i}(s) & -\tilde{B}_{i}(s) & -\tilde{C}_{i}(s) & -\tilde{D}_{i}(s) \\
\tilde{B}_{i}(s) & \tilde{A}_{i}(s) & -\tilde{D}_{i}(s) & \tilde{C}_{i}(s) \\
\tilde{C}_{i}(s) & \tilde{D}_{i}(s) & \tilde{A}_{i}(s) & -\tilde{B}_{i}(s) \\
\tilde{D}_{i}(s) & -\tilde{C}_{i}(s) & \tilde{B}_{i}(s) & \tilde{A}_{i}(s)
\end{array}\right] \otimes I_{n_{\mathrm{H}, i}}
$$

for the diagonal blocks corresponding to the $\rho_{\mathbb{H}, i}$ 's.

The notation may be further streamlined by using complex numbers in (9a) and (10c) and quaternions in (9b) and (10d). Coding a typical vector $\operatorname{col}\left(\tilde{w}_{\mathbb{C}, i}^{1}, \tilde{w}_{\mathbb{C}, i}^{2}\right) \in$ $\mathbb{R}^{2 n_{\mathbb{C}, i}}$ in $(9 \mathrm{a})$ as the complex vector $\tilde{w}_{\mathbb{C}, i}^{1}+i \tilde{w}_{\mathbb{C}, i}^{2} \in \mathbb{C}^{n_{\mathbb{C}, i}}$, ensures that multiplication by $(10 \mathrm{c})$ corresponds to multiplication by the complex polynomial matrix $\left(\tilde{A}_{i}(s)+\right.$ $\left.i \tilde{B}_{i}(s)\right) \otimes I_{n_{\mathbf{C}, i}}$. Coding a typical vector $\tilde{w}_{\mathbb{H}, i}=\operatorname{col}\left(\tilde{w}_{\mathbb{H}, i}^{1}, \tilde{w}_{\mathbb{H}, i}^{2}, \tilde{w}_{\mathbb{H}, i}^{3}, \tilde{w}_{\mathbb{H}, i}^{4}\right)$ in $(9 \mathrm{~b})$ as the quaternionic vector $\tilde{w}_{\mathbb{H}, i}^{1}+i \tilde{w}_{\mathbb{H}, i}^{2}+j \tilde{w}_{\mathbb{H}, i}^{3}+k \tilde{w}_{\mathbb{H}, i}^{4}$ ensures that multiplication by (10d) corresponds to multiplication by the quaternionic polynomial matrix $\left(\tilde{A}_{i}(s)+\right.$ $\left.i \tilde{B}_{i}(s)+j \tilde{C}_{i}(s)+k \tilde{D}_{i}(s)\right) \otimes I_{n_{\mathrm{H}, i}}$. For the multiplication rules for quaternions, see Example 8 and $\S 10$.

In the following theorem, we assume that the real $\rho$-adapted basis has been streamlined in this way. These considerations prove that, for $\mathbb{K}=\mathbb{R}$, Theorem 3 is equivalent to the following theorem.

THEOREM 5. Let $G$ be a compact group and let $\rho: G \rightarrow G L\left(\mathbb{R}^{q}\right)$ be a representation of $G$ on $\mathbb{R}^{q}$. Assume that $\rho$ is decomposed as (8) and that the basis in $\mathbb{R}^{q}$ is real $\rho$-adapted. To emphasize this, we write the signal variables as $\tilde{w}$,

$$
\begin{aligned}
& \tilde{w}=\operatorname{col}\left(\tilde{w}_{\mathbb{R}}, \tilde{w}_{\mathbb{C}}, \tilde{w}_{\mathbb{H}}\right), \\
& \tilde{w}_{\mathbb{R}}=\operatorname{col}\left(\tilde{w}_{\mathbb{R}, 1}, \tilde{w}_{\mathbb{R}, 2}, \ldots, \tilde{w}_{\mathbb{R}, k_{\mathbb{R}}}\right) \quad \text { with } \quad \tilde{w}_{\mathbb{R}, i}: \mathbb{R} \rightarrow\left(\mathbb{R}^{n_{\mathbb{R}, i}}\right)^{m_{\mathbb{R}, i}} \text {, } \\
& \tilde{w}_{\mathbb{C}}=\operatorname{col}\left(\tilde{w}_{\mathbb{C}, 1}, \tilde{w}_{\mathbb{C}, 2}, \ldots, \tilde{w}_{\mathbb{C}, k_{\mathbb{C}}}\right) \quad \text { with } \quad \tilde{w}_{\mathbb{C}, i}: \mathbb{R} \rightarrow\left(\mathbb{C}^{n_{\mathbb{C}, i}}\right)^{m_{\mathbb{C}, i}} \text {, } \\
& \tilde{w}_{\mathbb{H}}=\operatorname{col}\left(\tilde{w}_{\mathbb{H}, 1}, \tilde{w}_{\mathbb{H}, 2}, \ldots, \tilde{w}_{\mathbb{H}, k_{\mathbb{H}}}\right) \quad \text { with } \quad \tilde{w}_{\mathbb{H}, i}: \mathbb{R} \rightarrow\left(\mathbb{H}^{n_{\mathbb{H}, i}}\right)^{m_{\mathbb{H}, i}},
\end{aligned}
$$

as explained in the preamble.

Then $\Sigma=\left(\mathbb{R}, \mathbb{R}^{q}, \mathcal{B}\right) \in \mathcal{L}^{q}$ is $\rho$-symmetric if and only if there exist $m_{\mathbb{R}, i}^{\prime} \in \mathbb{Z}_{+}, 0 \leq$ $m_{\mathbb{R}, i}^{\prime} \leq m_{\mathbb{R}, i} ; m_{\mathbb{C}, i}^{\prime} \in \mathbb{Z}_{+}, 0 \leq m_{\mathbb{C}, i}^{\prime} \leq m_{\mathbb{C}, i} ; m_{\mathbb{H}, i}^{\prime} \in \mathbb{Z}_{+}, 0 \leq m_{\mathbb{H}, i}^{\prime} \leq m_{\mathbb{H}, i}$, and polynomial matrices $A_{i} \in \mathbb{R}_{f r}^{m_{\mathbb{R}, i}^{\prime} \times m_{\mathbb{R}, i}}[s], C_{i} \in \mathbb{C}_{f r}^{m_{\mathbb{C}, i}^{\prime} \times m_{\mathbb{C}, i}}[s], H_{i} \in \mathbb{H}_{f r}^{m_{H, i}^{\prime} \times m_{\mathbb{H}, i}}[s]$ such that $\Sigma$ admits a minimal representation

$$
\begin{array}{ll}
\left(A_{i}\left(\frac{d}{d t}\right) \otimes I_{n_{\mathbb{R}, i}}\right) \tilde{w}_{\mathbb{R}, i}=0, & i=1,2, \ldots, k_{\mathbb{R}}, \\
\left(C_{i}\left(\frac{d}{d t}\right) \otimes I_{n_{\mathbb{C}, i}}\right) \tilde{w}_{\mathbb{C}, i}=0, & i=1,2, \ldots, k_{\mathbb{C}}, \\
\left(H_{i}\left(\frac{d}{d t}\right) \otimes I_{n_{\mathbb{H}, i}}\right) \tilde{w}_{\mathbb{H}, i}=0, & i=1,2, \ldots, k_{\mathbb{H}} .
\end{array}
$$

\section{Applications.}

\subsection{Permutation symmetries.}

Example 4 (simple permutations). Our first class of examples all involve the symmetric group $S_{q}$ defined in Example 3, and we use the notation introduced there. 
Let us now apply Theorem 5 to a system $\Sigma=\left(\mathbb{R}, \mathbb{R}^{q}, \mathcal{B}\right) \in \mathcal{L}^{q}$ with $\rho: S_{q} \rightarrow G L\left(\mathbb{R}^{q}\right)$ where $\rho_{g} \operatorname{col}\left(w_{1}, w_{2}, \ldots, w_{q}\right):=\operatorname{col}\left(w_{g(1)}, w_{g(2)}, \ldots, w_{g(q)}\right)$. We obtain a suitable $\rho-$ adapted basis by taking as new coordinates $\tilde{x}_{1}:=x_{a v}, \tilde{x}_{2}:=\Delta x_{2}, \ldots, \tilde{x}_{q}:=\Delta x_{q}$, with $x_{a v}:=(1 / q)\left(x_{1}+x_{2}+\cdots+x_{q}\right)$ and $\Delta x_{i}=x_{i}-x_{a v}(i=1,2, \ldots, q)$. Clearly, $V_{1}=\left\{\operatorname{col}\left(\tilde{x}_{1}, \tilde{x}_{2}, \ldots, \tilde{x}_{q}\right) \in \mathbb{R}^{q} \mid \tilde{x}_{2}=\cdots=\tilde{x}_{q}=0\right\}$ and $V_{2}=\left\{\operatorname{col}\left(\tilde{x}_{1}, \tilde{x}_{2}, \ldots, \tilde{x}_{q}\right) \in\right.$ $\left.\mathbb{R}^{q} \mid \tilde{x}_{1}=0\right\}$. Observe that both the representations $\rho_{1}$ and $\rho_{2}$ introduced in Example 3 are of real type. Hence the decomposition $\rho \cong \rho_{1} \oplus \rho_{2}$ applies to the real case. It follows that, in terms of the notation of Theorem $5, m_{\mathbb{R}, 1}=m_{\mathbb{R}, 2}=1$ and that all the other multiplicities are zero. Furthermore, $n_{\mathbb{R}, 1}=1$ and $n_{\mathbb{R}, 2}=q-1$. Thus we must consider the following choices of $m_{\mathbb{R}, i}^{\prime}$ 's:

$$
\begin{gathered}
m_{\mathbb{R}, 1}^{\prime}=m_{\mathbb{R}, 2}^{\prime}=0, \\
m_{\mathbb{R}, 1}^{\prime}=1 ; \quad m_{\mathbb{R}, 2}^{\prime}=0, \\
m_{\mathbb{R}, 1}^{\prime}=0 ; \quad m_{\mathbb{R}, 2}^{\prime}=1, \\
m_{\mathbb{R}, 1}^{\prime}=m_{\mathbb{R}, 2}^{\prime}=1 .
\end{gathered}
$$

The first case corresponds to the trivial situation $\mathcal{B}=C^{\infty}\left(\mathbb{R}, \mathbb{R}^{q}\right)$. In the second case, $\rho$-symmetry corresponds to a minimal representation of the form

$$
r_{a v}\left(\frac{d}{d t}\right) w_{a v}=0
$$

with $w_{a v}:=(1 / q)\left(w_{1}+w_{2}+\cdots+w_{q}\right)$. This representation is determined by the nonzero polynomial $r_{a v} \in \mathbb{R}[s]$. The third case yields

$$
r_{\Delta}\left(\frac{d}{d t}\right) \Delta w_{i}=0, \quad i=2, \ldots, q
$$

with $\Delta w_{i}:=w_{i}-w_{a v}$. This representation is completely determined by the nonzero polynomial $r_{\Delta} \in \mathbb{R}[s]$. Note that these equations imply the redundant equation $r_{\Delta}(d / d t) \Delta w_{1}=0$, and hence, by letting the above equation range over $i=1,2, \ldots, q$, we obtain an equivalent but not minimal representation. In the fourth case, we will obtain one equation on $w_{a v}$ and one on each of the $\Delta w_{i}$ 's, and these equations are all identical.

It is clear that, by allowing nonminimal representations, all four cases can be captured in one. It follows that $\Sigma \in \mathcal{L}^{q}$ will be $\rho$-symmetric in the case of these simple permutations if and only if there exist (not necessarily nonzero) polynomials $r_{a v} \in \mathbb{R}[s], r_{\Delta} \in \mathbb{R}[s]$ such that $\Sigma$ is described by

$$
\begin{aligned}
r_{a v}\left(\frac{d}{d t}\right) w_{a v} & =0 \\
r_{\Delta}\left(\frac{d}{d t}\right) \Delta w_{i}=0, \quad i & =1,2, \ldots, q
\end{aligned}
$$

with $w_{a v}:=(1 / q)\left(w_{1}+w_{2}+\cdots+w_{q}\right)$ and $\Delta w_{i}:=w_{i}-w_{a v}$. Hence a symmetric system is governed by two equations. One equation governs the dynamics of the average (consider it an equation governing the center of mass in the case of motion of identical particles on the line). The second equation is identical for each of the components and governs the dynamics of the distance from the average and is identical for each of the components (consider this equation as governing the motion of the displacement of the particle from the center of mass). Note that either one or both of these equations 
may be absent (when $r_{a v}=0$ and/or $r_{\Delta}=0$ ). The most important feature of the above equations is the fact that the different variables $w_{i}$ interact only through their average value. For an analogue nonlinear situation of this example, see [17].

Example 5 (permutations of identical subsystems with feature vectors). Next, consider the system $\Sigma=\left(\mathbb{R},\left(\mathbb{R}^{m}\right)^{n}, \mathcal{B}\right) \in \mathcal{L}^{m n}$. Think of $\Sigma$ as modelling $n$ identical subsystems, each of which is described by $m$ features. Thus $w=\operatorname{col}\left(w_{1}, w_{2}, \ldots, w_{n}\right)$ with each of the $w_{i}: \mathbb{R} \rightarrow \mathbb{R}^{m}, i=1,2, \ldots, n$, where $w_{i}(t) \in \mathbb{R}^{m}$ denotes the feature vector of the $i$ th subsystem at time $t$. In the case of the motion of $n$ particles, this feature vector could be the position of the particle in the plane $(m=2)$ or in 3-space $(m=3)$, or we could consider each particle being described by a position and an external force acting on it (thus $m=2,4$, or 6 , depending on whether these particles are considered on the line, in the plane, or in 3-space).

Let $\rho: S_{n} \rightarrow G L\left(\left(\mathbb{R}^{m}\right)^{n}\right)$ act as follows:

$$
\rho_{g} \operatorname{col}\left(w_{1}, w_{2}, \ldots, w_{n}\right):=\operatorname{col}\left(w_{g(1)}, w_{g(2)}, \ldots, w_{g(n)}\right) .
$$

In this case, the decomposition of $\rho$ into irreducible components leads to $\rho \cong m \rho_{1} \oplus$ $m \rho_{2}$ with $\rho_{1}$ and $\rho_{2}$ as in Example 3 or Example 4 . The $\rho$-adapted basis may now be chosen as follows. Define, for $x=\operatorname{col}\left(x_{1}, x_{2}, \ldots, x_{n}\right) \in\left(\mathbb{R}^{m}\right)^{n}, x_{a v}:=$ $(1 / n)\left(x_{1}+x_{2}+\cdots+x_{n}\right)$ and $\Delta x_{i}:=x_{i}-x_{a v}$. Represent $x$ by the coordinate vector $\operatorname{col}\left(x_{a v}, \Delta x_{2}, \ldots, \Delta x_{n}\right)$ and define $V_{1}$ by $\Delta x_{2}=\cdots=\Delta x_{n}=0$ and $V_{2}$ by $x_{a v}=0$. Then $\left.m \rho_{1} \cong \rho\right|_{V_{1}}$ and $\left.m \rho_{2} \cong \rho\right|_{V_{2}}$. The further decomposition of $m \rho_{1}$ and $m \rho_{2}$ into their irreducible components is rather obvious but will not be given, since it will not be needed in the following.

In terms of the notation of Theorem 5 , we have $m_{\mathbb{R}, 1}=m_{\mathbb{R}, 2}=m$ and $n_{\mathbb{R}, 1}=$ $1, n_{\mathbb{R}, 2}=n-1$. Thus we should consider all the cases where $0 \leq m_{\mathbb{R}, 2}^{\prime}, m_{\mathbb{R}, 2}^{\prime} \leq m$. Proceeding exactly as in Example 4 we obtain that a system $\Sigma=\left(\mathbb{R},\left(\mathbb{R}^{m}\right)^{n}, \mathcal{B}\right) \in \mathcal{L}^{m n}$ will be $\rho$-symmetric if and only if there exist $m_{a v}, m_{\Delta} \in \mathbb{Z}_{+}$(we could, but need not, restrict $m_{a v}$ and $m_{\Delta}$ to be $\left.\leq m\right)$ and polynomial matrices $R_{a v} \in \mathbb{R}^{m_{a v} \times m}[s]$ and $R_{\Delta} \in \mathbb{R}^{m_{\Delta} \times m}[s]$ such that $\Sigma$ is described by

$$
\begin{gathered}
R_{a v}\left(\frac{d}{d t}\right) w_{a v}=0 \\
R_{\Delta}\left(\frac{d}{d t}\right) \Delta w_{i}=0, \quad i=1,2, \ldots, n
\end{gathered}
$$

with $w_{a v}:=(1 / n)\left(w_{1}+w_{2}+\cdots+w_{n}\right)$ and $\Delta w_{i}:=w_{i}-w_{a v}$.

As a more specific example, consider a system of $n$ identical particles in 3-space with external forces. Such a system will hence be described by differential equations of the form

$$
\begin{gathered}
P_{a v}\left(\frac{d}{d t}\right) q_{a v}=Q_{a v}\left(\frac{d}{d t}\right) F_{a v} \\
P_{\Delta}\left(\frac{d}{d t}\right)\left(q_{i}-q_{a v}\right)=Q_{\Delta}\left(\frac{d}{d t}\right)\left(F_{i}-F_{a v}\right), \quad i=1,2, \ldots, n
\end{gathered}
$$

with $q_{i}$ the position of the $i$ th particle, $F_{i}$ the external force acting on it, and $q_{a v}, F_{a v}$ defined in the obvious way. Thus the motion of the center of mass is governed by a law involving the mean force, while the laws governing the motion of the displacement from the center of mass involves the difference of the force acting on the particle and 
the mean force and is identical for each of the particles. In particular, the particles interact only through the center of mass and the mean force.

Example 6 (permutations with two kinds of subsystems). Now consider the system

$$
\Sigma=\left(\mathbb{R},\left(\mathbb{R}^{m_{1}}\right)^{n_{1}} \times\left(\mathbb{R}^{m_{2}}\right)^{n_{2}}, \mathcal{B}\right) \in \mathcal{L}^{m_{1} n_{1}+m_{2} n_{2}} .
$$

Think of $\Sigma$ as modelling $n_{1}$ identical subsystems of one kind with each $m_{1}$ features and $n_{2}$ identical subsystems of a second kind each with $m_{2}$ features.

Let $\rho: S_{n_{1}} \times S_{n_{2}} \rightarrow G L\left(\left(\mathbb{R}^{m_{1}}\right)^{n_{1}} \times\left(\mathbb{R}^{m_{2}}\right)^{n_{2}}\right)$ act as follows:

$$
\rho_{g_{1}, g_{2}} \operatorname{col}\left(w_{1}^{\prime}, \ldots, w_{n_{1}}^{\prime}, w_{1}^{\prime \prime}, \ldots, w_{n_{2}}^{\prime \prime}\right):=\operatorname{col}\left(w_{g_{1}(1)}^{\prime}, \ldots, w_{g_{1}\left(n_{1}\right)}^{\prime}, w_{g_{2}(1)}^{\prime \prime}, \ldots, w_{g_{2}\left(n_{2}\right)}^{\prime \prime}\right) \text {. }
$$

The decomposition of $\rho$ into irreducible components now becomes $\rho \cong\left(m_{1}+m_{2}\right) \rho_{1} \oplus$ $m_{1} \rho_{2}^{\prime} \oplus m_{2} \rho_{2}^{\prime \prime}$ with $\rho_{1}$ the identity representation and $\left.\left(m_{1}+m_{2}\right) \rho_{1} \cong \rho\right|_{V_{1}}$ with

$$
V_{1}=\left\{\operatorname{col}\left(x_{1}^{\prime}, \ldots x_{n_{1}}^{\prime}, x_{1}^{\prime \prime}, \ldots, x_{n_{2}}^{\prime \prime}\right) \mid x_{1}^{\prime}=\cdots=x_{n_{1}}^{\prime}, x_{1}^{\prime \prime}=\cdots=x_{n_{2}}^{\prime \prime}\right\} .
$$

Furthermore, $\rho_{2}^{\prime}$ and $\rho_{2}^{\prime \prime}$ are the analogues of what we denoted by $\rho_{2}$ before: $\rho_{2}^{\prime}$ corresponds to the analogue of $\rho_{2}$ as an irreducible representation of $S_{n_{1}}$ and $\rho_{2}^{\prime \prime}$ corresponds to the analogue of $\rho_{2}$ as an irreducible representation of $S_{n_{2}}$. Proceeding as before, Theorem 5 will show that $\Sigma$ is $\rho$-symmetric if and only if there exist $m_{a v}, m_{\Delta}^{\prime}, m_{\Delta}^{\prime \prime} \in \mathbb{Z}_{+}$and polynomial matrices $R_{a v} \in \mathbb{R}^{m_{a v} \times\left(m_{1}+m_{2}\right)}[s], R_{\Delta}^{\prime} \in$ $\mathbb{R}^{m_{\Delta}^{\prime} \times m_{1}}[s]$, and $R_{\Delta}^{\prime \prime} \in \mathbb{R}^{m_{\Delta}^{\prime \prime} \times m_{2}}[s]$ such that $\Sigma$ is described by

$$
\begin{gathered}
R_{a v}\left(\frac{d}{d t}\right)\left[\begin{array}{c}
w_{a v}^{\prime} \\
\cdots \cdots \\
w_{a v}^{\prime \prime}
\end{array}\right]=0, \\
R_{\Delta}^{\prime}\left(\frac{d}{d t}\right)\left(w_{i}^{\prime}-w_{a v}^{\prime}\right)=0, \quad i=1,2, \ldots, n_{1}, \\
R_{\Delta}^{\prime \prime}\left(\frac{d}{d t}\right)\left(w_{j}^{\prime \prime}-w_{a v}^{\prime \prime}\right)=0, \quad j=1,2, \ldots, n_{2} .
\end{gathered}
$$

This shows that the two groups can only interact through their averages, while all the respective displacements are independent.

An interesting special case can now be obtained by taking $n_{2}=1$. We can then view the situation as modelling the dynamics of the interaction of a central control station with $n$ identical substations. In the obvious notation, the dynamical laws then take the form

$$
\begin{gathered}
R_{a v}\left(\frac{d}{d t}\right)\left[\begin{array}{l}
w_{a v} \\
\cdots \ldots . \\
w_{\text {central }}
\end{array}\right]=0, \\
R_{\Delta}\left(\frac{d}{d t}\right)\left(w_{i}-w_{a v}\right)=0, \quad i=1,2, \ldots, n .
\end{gathered}
$$

Thus the central controller influences only the average of the feature vectors of the substations.

Remark. The above examples all involve the action of the whole symmetric group $S_{n}$. Actually, identical results may be obtained by considering a doubly transitive subgroup $G$ of $S_{n}$. A subgroup $G \subseteq S_{n}$ is said to be transitive if, for all $\alpha, \beta \in$ $\{1,2, \ldots, n\}$, there exist $g \in G$ such that $g(\alpha)=\beta$; it is said to be doubly transitive 
if, for all $\alpha^{\prime}, \beta^{\prime}, \alpha^{\prime \prime}, \beta^{\prime \prime}, \in\{1,2, \ldots, n\}, \alpha^{\prime} \neq \alpha^{\prime \prime}$, and $\beta^{\prime} \neq \beta^{\prime \prime}$, there exists $g \in G$ such that $g\left(\alpha^{\prime}\right)=\beta^{\prime}$ and $g\left(\alpha^{\prime \prime}\right)=\beta^{\prime \prime}$. An example of a doubly transitive subgroup is the subgroup of even permutations.

It can be shown that the representations of Examples 4-6 remain valid without changes if we assume invariance of $\mathcal{B}$ for a doubly transitive subgroup $G$ of $S_{n}$ in Example 5 and doubly transitive subgroups $G_{1}$ of $S_{n_{1}}$ and $G_{2}$ of $S_{n_{2}}$ in Example 6. In particular, this shows that permutation symmetry for one doubly transitive subgroup implies permutation symmetry for the whole of $S_{n}$ in Example 5 and analogously for the whole of $S_{n_{1}} \times S_{n_{2}}$ in Example 6!

Example 7 (cyclic permutations). Let $\mathbb{Z}_{q}$ denote the group consisting of

$$
\{0,1, \ldots, q-1\}
$$

with the group operation addition modulo $q$. It is more convenient to denote this group as $\left\{1, r, r^{2}, \ldots, r^{q-1}\right\}$ with $r^{q}=1$. Note that $\mathbb{Z}_{q}$ is a subgroup of $S_{q}$. It is called the group of cyclic permutations. Let $\mathbb{Z}_{q}=\left\{1, r, \ldots, r^{q-1}\right\}$ act on $\mathbb{K}^{q}$ as in the case of permutations. Thus

$$
\rho_{r} \operatorname{col}\left(x_{1}, x_{2}, \ldots, x_{q-1}, x_{q}\right):=\operatorname{col}\left(x_{q}, x_{1}, x_{2}, \ldots, x_{q-1}\right)
$$

from which $\rho_{r}, \ldots, \rho_{r^{q-1}}$ follow.

Because the group $\mathbb{Z}_{q}$ is commutative, its irreducible representations over $\mathbb{C}$ are all one-dimensional. There are $q$ such irreducible representations given by $\rho_{1}, \rho_{2}, \ldots, \rho_{q}$ with $\rho_{k}: \mathbb{Z}_{q} \rightarrow G L(\mathbb{C})$ given by $\rho_{k, r}=\lambda^{k}$ with $\lambda:=e^{i(2 \pi / q)}$. A simple calculation shows that $\rho \cong \rho_{1} \oplus \rho_{2} \oplus \cdots \oplus \rho_{q}$, with the invariant subspace corresponding to $\rho_{k}$ given by span $\operatorname{col}\left(1, \lambda^{-k}, \lambda^{-2 k}, \ldots, \lambda^{-(q-1) k}\right)$. This now allows us to compute the $\rho$-adapted basis. We omit the detailed calculations.

Theorem 4 implies that $\Sigma=\left(\mathbb{R}, \mathbb{R}^{q}, \mathcal{B}\right) \in \mathcal{L}^{q}$ will be symmetric with respect to the cyclic permutations if and only if there exist polynomials $r_{1}, r_{2}, \ldots, r_{q} \in \mathbb{C}[z]$, $p(\Sigma)$ of which are nonzero, such that

$$
r_{j}\left(\frac{d}{d t}\right)\left(\sum_{k=1}^{q} \lambda^{-j k} w_{k}\right)=0, \quad j=1,2, \ldots, q
$$

forms a minimal representation of $\Sigma$. This set of equations can be edited a bit further and leads to the following canonical form for this cyclic symmetry. $\Sigma$ will be symmetric if and only if there exist polynomials $\tilde{r}_{1}, \tilde{r}_{2}, \ldots, \tilde{r}_{q} \in \mathbb{C}[s]$ and a representation of $\Sigma$ of the following form:

$$
\left[\begin{array}{ccccc}
\tilde{r}_{1}\left(\frac{d}{d t}\right) & \tilde{r}_{2}\left(\frac{d}{d t}\right) & \cdots & \tilde{r}_{q-1}\left(\frac{d}{d t}\right) & \tilde{r}_{q}\left(\frac{d}{d t}\right) \\
\tilde{r}_{q}\left(\frac{d}{d t}\right) & \tilde{r}_{1}\left(\frac{d}{d t}\right) & \cdots & \tilde{r}_{q-2}\left(\frac{d}{d t}\right) & \tilde{r}_{q-1}\left(\frac{d}{d t}\right) \\
\vdots & \vdots & \ddots & \vdots & \vdots \\
\tilde{r}_{2}\left(\frac{d}{d t}\right) & \tilde{r}_{3}\left(\frac{d}{d t}\right) & \cdots & \tilde{r}_{q}\left(\frac{d}{d t}\right) & \tilde{r}_{1}\left(\frac{d}{d t}\right)
\end{array}\right]\left[\begin{array}{c}
w_{1} \\
w_{2} \\
\vdots \\
w_{q}
\end{array}\right]=0 .
$$

Note that this representation (which is possibly nonminimal) puts the cyclic symmetry nicely into evidence.

In the real case where $\mathbb{K}=\mathbb{R}$, we must combine the complex conjugate irreducible representations. Similar calculations to the above ones lead to (possibly nonminimal) 
behavioral equations

$$
\begin{gathered}
r_{0}\left(\frac{d}{d t}\right)\left(\sum_{k=1}^{q} w_{k}\right)=0 \\
r_{j}^{\prime}\left(\frac{d}{d t}\right)\left(\sum_{k=1}^{q} w_{k} \cos \frac{2 \pi j}{q} k\right)+r_{j}^{\prime \prime}\left(\frac{d}{d t}\right)\left(\sum_{k=1}^{q} w_{k} \sin \frac{2 \pi j}{q} k\right)=0 \\
r_{j}^{\prime \prime}\left(\frac{d}{d t}\right)\left(\sum_{k=1}^{q} w_{k} \cos \frac{2 \pi j}{q} k\right)-r_{j}^{\prime}\left(\frac{d}{d t}\right)\left(\sum_{k=1}^{q} w_{k} \sin \frac{2 \pi j}{q} k\right)=0, \\
\text { for } j=1,2, \frac{q-1}{2}, \quad \text { if } q \text { is odd, } \\
\text { for } j=1,2, \ldots, \frac{q}{2}-1, \quad \text { if } q \text { is even. }
\end{gathered}
$$

In the case that $q$ is even, there is an additional equation

$$
r_{\frac{q}{2}}\left(\frac{d}{d t}\right)\left(\sum_{k=1}^{q}(-1)^{k} w_{k}\right)=0
$$

where $r_{0}, r_{j}^{\prime}, r_{j}^{\prime \prime}, r_{q / 2} \in \mathbb{R}[s]$.

The above calculations are easily generalized to cyclic permutation symmetries with feature vectors. In this case, it suffices to interpret the $r$ 's as matrices.

\subsection{A quaternionic symmetry.}

Example 8. Consider $\left(\mathbb{R}, \mathbb{R}^{q}, \mathcal{B}\right) \in \mathcal{L}^{q}$ with $q=4$. Now assume that this system is symmetric in the following sense:

$$
\left(\left[\begin{array}{l}
w_{1} \\
w_{2} \\
w_{3} \\
w_{4}
\end{array}\right] \in \mathcal{B}\right) \Rightarrow\left(\left[\begin{array}{r}
-w_{2} \\
w_{1} \\
-w_{4} \\
w_{3}
\end{array}\right],\left[\begin{array}{r}
-w_{3} \\
w_{4} \\
w_{1} \\
-w_{2}
\end{array}\right],\left[\begin{array}{r}
-w_{4} \\
-w_{3} \\
w_{2} \\
w_{1}
\end{array}\right] \in \mathcal{B}\right)
$$

As we will see, this is a quaternionic symmetry. We will not give a physical example where such a symmetry can occur.

The group of quaternions consists of $\mathcal{H}=\{ \pm 1, \pm i, \pm j, \pm k\}$ with multiplication table

$$
i^{2}=j^{2}=k^{2}=-1, i j=-j i=k, j k=-k j=i, k i=-i k=-j .
$$

The following defines a representation of $\mathcal{H}$ on $\mathbb{C}^{2}$

$$
\begin{array}{ll} 
\pm 1 \mapsto \pm\left[\begin{array}{cc}
1 & 0 \\
0 & 1
\end{array}\right], & \pm i \mapsto \pm\left[\begin{array}{rr}
0 & -1 \\
1 & 0
\end{array}\right], \\
\pm j \mapsto \pm\left[\begin{array}{rr}
i & 0 \\
0 & -i
\end{array}\right], & \pm k \mapsto \pm\left[\begin{array}{ll}
0 & i \\
i & 0
\end{array}\right] .
\end{array}
$$

This representation is irreducible. It is obviously not real and, since all the above matrices have real trace, it is a complex representation of quaternionic type. The 
representation induced on $\mathbb{R}^{4}$ by combining this representation with its complex conjugate yields

$$
\begin{aligned}
1 \mapsto\left[\begin{array}{rrrr}
1 & 0 & 0 & 0 \\
0 & 1 & 0 & 0 \\
0 & 0 & 1 & 0 \\
0 & 0 & 0 & 1
\end{array}\right], \quad i \mapsto M_{1}:=\left[\begin{array}{rrrr}
0 & -1 & 0 & 0 \\
1 & 0 & 0 & 0 \\
0 & 0 & 0 & -1 \\
0 & 0 & 1 & 0
\end{array}\right], \\
j \mapsto M_{2}:=\left[\begin{array}{rrrr}
0 & 0 & -1 & 0 \\
0 & 0 & 0 & 1 \\
1 & 0 & 0 & 0 \\
0 & -1 & 0 & 0
\end{array}\right], \quad k \mapsto M_{3}:=\left[\begin{array}{rrrr}
0 & 0 & 0 & -1 \\
0 & 0 & -1 & 0 \\
0 & 1 & 0 & 0 \\
1 & 0 & 0 & 0
\end{array}\right] .
\end{aligned}
$$

Note that $M_{1}, M_{2}, M_{3}$ are precisely the matrices defining the static symmetry under consideration.

It follows from Theorem 5 that the $\Sigma$ 's that are symmetric in this sense are precisely those that admit a representation for the form

$$
\begin{aligned}
& a\left(\frac{d}{d t}\right) w_{1}-b\left(\frac{d}{d t}\right) w_{2}-c\left(\frac{d}{d t}\right) w_{3}-d\left(\frac{d}{d t}\right) w_{4}=0 \\
& b\left(\frac{d}{d t}\right) w_{1}+a\left(\frac{d}{d t}\right) w_{2}+d\left(\frac{d}{d t}\right) w_{3}-c\left(\frac{d}{d t}\right) w_{4}=0 \\
& c\left(\frac{d}{d t}\right) w_{1}-d\left(\frac{d}{d t}\right) w_{2}+a\left(\frac{d}{d t}\right) w_{3}+b\left(\frac{d}{d t}\right) w_{4}=0 \\
& d\left(\frac{d}{d t}\right) w_{1}+c\left(\frac{d}{d t}\right) w_{2}-b\left(\frac{d}{d t}\right) w_{3}+a\left(\frac{d}{d t}\right) w_{4}=0
\end{aligned}
$$

with $a, b, c, d \in \mathbb{R}[s]$.

7.3. Symmetries with Lie groups. A Lie group $G$ is a topological group with the structure of a $C^{\infty}$ differentiable $\mathbb{K}$-manifold in which the group multiplication and the inverse are $C^{\infty}$ maps. A $C^{\infty}$ group homomorphism $\rho: G \rightarrow G L\left(\mathbb{K}^{n}\right)$ is said to be a representation of the Lie group $G$ on $\mathbb{K}^{n}$. Let $g \ell\left(\mathbb{K}^{n}\right)$ denote the set of $n \times n$ matrices over $\mathbb{K}$ endowed with the usual commutator product $[A, B]=A B-B A ; g \ell\left(\mathbb{K}^{n}\right)$ is the Lie algebra of $G L\left(\mathbb{K}^{n}\right)$. Let $\mathcal{G}$ be the Lie algebra of $G$. The representation $\rho: G \rightarrow G L\left(\mathbb{K}^{n}\right)$ of the Lie group $G$ on $\mathbb{K}^{n}$ induces a Lie algebra homomorphism $\tilde{\rho}: \mathcal{G} \rightarrow g \ell\left(\mathbb{K}^{n}\right)$ of the Lie algebra $\mathcal{G}$ on $\mathbb{K}^{n}$. Let $X_{1}, X_{2}, \ldots, X_{N} \in \mathbb{K}^{n \times n}$ be a set of generators of $\tilde{\rho}(\mathcal{G})$. Then we have the following result.

THEOREM 6. Let $\mathcal{G}$ be a compact connected Lie group and let $\rho: G \rightarrow G L\left(\mathbb{K}^{q}\right)$ be a representation of the Lie group $\mathcal{G}$ on $\mathbb{K}^{q}$. Let $X_{1}, X_{2}, \ldots, X_{N} \in \mathbb{K}^{q \times q}$ be a set of generators of $\tilde{\rho}(\mathcal{G})$ with $\tilde{\rho}$ the induced representation of the Lie algebra $\mathcal{G}$ on $\mathbb{K}^{q}$. Then the following are equivalent for $\Sigma=\left(\mathbb{R}, \mathbb{K}^{q}, \mathcal{B}\right) \in \mathcal{L}^{q}$ :

(1) $\Sigma$ is $\rho$-symmetric;

(2) $X \mathcal{B} \subseteq \mathcal{B}$ for all $X \in \tilde{\rho}(\mathcal{G})$;

(3) $X_{i} \mathcal{B} \subseteq \mathcal{B}$ for $i=1,2, \ldots, N$

(4) There exist matrices $Y_{1}, Y_{2}, \ldots, Y_{n} \in \mathbb{K}^{p(\Sigma) \times p(\Sigma)}$ and a $R \in \mathbb{K}^{p(\Sigma) \times q}[s]$ such that $R(d / d t) w=0$ is a minimal representation for $\Sigma$ with $Y_{i} R(s)=R(s) X_{i}$ for $i=1,2, \ldots, N$. Moreover, the subspace generated by the $Y_{i}$ 's is a Lie subalgebra of $g \ell\left(\mathbb{K}^{p(\Sigma)}\right)$ and yields through the association $X_{i} \mapsto Y_{i}$ a subrepresentation of the Lie algebra representation $\tilde{\rho}: \mathcal{G} \rightarrow g \ell\left(\mathbb{K}^{n}\right)$. 
Proof. Assume without loss of generality that $G$ is a subgroup of $G L\left(\mathbb{K}^{q}\right)$ and that $\rho$ is the canonical injection.

We will run the circle $(4) \Rightarrow(3) \Rightarrow(2) \Rightarrow(1) \Rightarrow(4)$. The first two implications are trivial.

To show that $(2) \Rightarrow(1)$, assume that $X \in \mathcal{G}$ and consider the exponential $\exp X$. It is well known that there exist $\alpha_{1}, \alpha_{2}, \ldots, \alpha_{n} \in \mathbb{K}$ such that $\exp X=\sum_{i=1}^{n} \alpha_{i} X^{i}$. This implies, by (2), that $(\exp X) \mathcal{B} \subseteq \mathcal{B}$. Since $G$ is compact connected, exp : $\mathcal{G} \rightarrow G$ is surjective, and hence (1) follows.

$(1) \Rightarrow(4)$ : From Theorem 3 , it follows that there exist $R \in \mathbb{K}^{p(\Sigma) \times q}[s]$ and a representation $\rho^{\prime}: G \rightarrow G L\left(\mathbb{K}^{p(\Sigma)}\right)$ such that $\rho^{\prime} R(s)=R(s) \rho$. Now consider the one-parameter subgroup of $G$ given by $G_{i}:=\left\{\exp \mu X_{i} \mid \mu \in \mathbb{K}\right\}$. Then $\rho^{\prime}\left(G_{i}\right)$ is also a one-parameter subgroup of $G L\left(\mathbb{K}^{p(\Sigma)}\right)$. Hence there exists a $Y_{i} \in g \ell\left(\mathbb{K}^{p(\Sigma)}\right)$ such that $\rho^{\prime}\left(\exp \mu X_{i}\right)=\exp \mu Y_{i}$. Hence $\left(\exp \mu Y_{i}\right) R(s)=R(s)\left(\exp \mu X_{i}\right)$. Differentiating at $\mu=0$ yields $Y_{i} R(s)=R(s) X_{i}$. The last part of (4) follows from the observation that $R(s) X_{i} X_{j}=Y_{i} R(s) X_{j}=Y_{i} Y_{j} R(s)$. Hence $R(s)\left[X_{i}, X_{j}\right]=\left[Y_{i}, Y_{j}\right] R(s)$. This shows that the vector space generated by these $Y_{i}$ 's is a Lie subalgebra of $g \ell\left(\mathbb{K}^{p(\Sigma)}\right)$ and that $X_{i} \mapsto Y_{i}$ generates a Lie algebra homomorphism.

\subsection{Rotation symmetries.}

Example 9 (rotations on $\mathbb{R}^{m}$ with $m>2$ ). Consider the group $S O(m)$ of real orthogonal $m \times m$ matrices with determinant 1 . $S O(m)$ is a compact group, and it can be shown that, when $m \geq 3$, the canonical injection of $S O(m)$ into $G L\left(\mathbb{C}^{m}\right)$ (which will also be denoted as $S O(m)$ ) is irreducible over $\mathbb{C}$.

Now consider the system $\Sigma=\left(\mathbb{R},\left(\mathbb{R}^{m}\right)^{n}, \mathcal{B}\right)$. Assume that $S O(m)$ acts on $\left(\mathbb{R}^{m}\right)^{n}$ by $M \in S O(m)$, taking $\operatorname{col}\left(w_{1}, w_{2}, \ldots, w_{n}\right)$ into $\operatorname{col}\left(M w_{1}, M w_{2}, \ldots, M w_{n}\right)$. Now consider the static symmetry induced by this action. To interpret this situation physically, think, for example, of $m=3$ and of $\Sigma$ as modelling the motion of $n$ (not identical) particles in $\mathbb{R}^{3}$ under the influence of rotation invariant laws. Another possibility is to think of the situation $m=3$ and $n=2 n^{\prime}$ with $\Sigma$ modelling the position and the force acting on $n^{\prime}$ particles.

Applying Theorem 5 and using the irreducibility of $S O(m)$ for $m>2$ immediately shows that $\Sigma$ will be symmetric if and only if there exists a polynomial matrix $R^{\prime} \in$ $\mathbb{R}^{\bullet \times n}[s]$ such that $\Sigma$ is represented as

$$
\left(R^{\prime}\left(\frac{d}{d t}\right) \otimes I_{m}\right)\left[\begin{array}{c}
w_{1} \\
w_{2} \\
\vdots \\
w_{n}
\end{array}\right]=0 .
$$

Example 10 (rotations on $\mathbb{R}^{2}$ ). The above example must be modified in the case where $m=2$, since the canonical injection of $S O(2)$ into $G L\left(\mathbb{C}^{2}\right)$ is reducible over $\mathbb{C}$. $S O(2)$ can be written as $S O(2) \cong \rho \oplus \rho^{*}$ with

$$
\rho\left(\left[\begin{array}{ll}
\cos \theta & -\sin \theta \\
\sin \theta & \cos \theta
\end{array}\right]\right)=e^{i \theta}
$$

Applying Theorem 5 leads to the following representation of rotation symmetric systems for $m=2$ :

$$
\left[\begin{array}{cc}
R_{1}\left(\frac{d}{d t}\right) & -R_{2}\left(\frac{d}{d t}\right) \\
R_{2}\left(\frac{d}{d t}\right) & R_{1}\left(\frac{d}{d t}\right)
\end{array}\right]\left[\begin{array}{l}
w_{1} \\
w_{2}
\end{array}\right]=0
$$


with $R_{1}, R_{2} \in \mathbb{R}^{(1 / 2) p(\Sigma) \times n}$.

This result can also be obtained from Theorem 6 . Indeed, since $\left[\begin{array}{cc}0 & 1 \\ -1 & 0\end{array}\right]$ generates $S O(2)$ through exponentiation, symmetry implies the existence of an $R$ such that

$$
\left[\begin{array}{rr}
0_{(1 / 2) p(\Sigma)} & I_{(1 / 2) p(\Sigma)} \\
-I_{(1 / 2) p(\Sigma)} & 0_{(1 / 2) p(\Sigma)}
\end{array}\right] R(s)=R(s)\left[\begin{array}{rr}
0_{n} & I_{n} \\
-I_{n} & 0_{n}
\end{array}\right],
$$

where $I_{k}$ and $O_{k}$ denote the $k \times k$ identity and zero matrices, respectively. This also yields the above representation.

Finally, systems with this rotation symmetry can also be represented by introducing the complex signal $w:=w_{1}+i w_{2}$ with $w: \mathbb{R} \rightarrow \mathbb{C}$. The differential equation governing

$$
\left[\begin{array}{l}
w_{1} \\
w_{2}
\end{array}\right]
$$

then becomes $R(d / d t) w=0$ with $R \in \mathbb{C}^{(1 / 2) p(\Sigma) \times n}[s]$.

Example 11 (rotations over $2 \pi / n$ degrees on $\mathbb{R}^{2}$ ). Let $G=\mathbb{Z}_{n}=\{0,1$, $\ldots, n-1\}$ (see Example 7 for notation) with $n \geq 3$. Now consider the representation $\rho$ of $G$ on $\mathbb{R}^{2}$, defined by

$$
\rho: k \mapsto\left[\begin{array}{rr}
\cos \frac{2 \pi k}{n} & -\sin \frac{2 \pi k}{n} \\
\sin \frac{2 \pi k}{n} & \cos \frac{2 \pi k}{n}
\end{array}\right]
$$

Then $\left(\mathbb{R}, \mathbb{R}^{2}, \mathcal{B}\right) \in \mathcal{L}^{2}$ being $\rho$-symmetric means symmetry in the sense of Example 10 , by rotations of $2 \pi / n$ degrees. This is a subgroup of $S O(2)$. Since the representation $\rho$ is also irreducible, we immediately obtain from Theorem 5 the representation obtained in Example 10. This allows the interesting conclusion that $\Sigma \in \mathcal{L}^{2}$ being symmetric with respect to rotations of $2 \pi / n$ degrees will imply that it is symmetric with respect to all rotations. The Lie algebra line of reasoning in Example 10 showed this already for $n=4$, that is, for $90^{\circ}$ rotations.

8. State space models. Many useful models encountered in applications involve auxiliary variables in addition to the variables that the model aims at describing. To distinguish between these two kinds of variables, we call the variables of primary interest manifest and denote them as $w$, and we call the auxiliary variables latent, and-usually-denote them as $\ell$.

Proceeding with the terminology of [15], this leads to a dynamical system with latent variables, defined as $\Sigma_{f}=\left(\mathbb{T}, \mathbb{W}, \mathbb{L}, \mathcal{B}_{f}\right)$ with $\mathbb{T} \subseteq \mathbb{R}$ the time axis, $\mathbb{W}$ the signal space of manifest variables, $\mathbb{L}$ the signal space of latent variables, and $\mathcal{B}_{f} \subseteq(\mathbb{W} \times \mathbb{L})^{\mathbb{T}}$ the full behavior. $\Sigma_{f}$ induces the manifest dynamical system $\Sigma=(\mathbb{T}, \mathbb{W}, \mathcal{B})$ with manifest behavior $\mathcal{B}=\left\{w \mid\right.$ there exists an $\ell$ such that $\left.(w, \ell) \in \mathcal{B}_{f}\right\}$.

In the context of systems described by differential equations, this leads us to consider linear differential equations

$$
R\left(\frac{d}{d t}\right) w=M\left(\frac{d}{d t}\right) \ell
$$

linking the manifest variables $w \in C^{\infty}\left(\mathbb{R}, \mathbb{K}^{q}\right)$ to the latent variables $\ell \in C^{\infty}\left(\mathbb{R}, \mathbb{K}^{d}\right)$. Here $R \in \mathbb{K}^{\bullet \times q}[s]$ and $M \in \mathbb{K}^{\bullet \times d}[s]$ are two polynomial matrices with the same number of rows. Formally, (12) defines the latent variable dynamical system $\left(\mathbb{R}, \mathbb{K}^{q}, \mathbb{K}^{d}, \mathcal{B}_{f}\right)$ 
with $\mathcal{B}_{f}=\operatorname{ker}[R(d / d t) \vdots-M(d / d t)]$. Obviously, $\left(\mathbb{R}, \mathbb{K}^{q+d}, \mathcal{B}_{f}\right) \in \mathcal{L}^{q+d}$. We will denote this class of latent variable dynamical systems as $\mathcal{L}^{q, d}$. This family of models is hence parametrized by pairs of polynomial matrices $(R, M)$.

This model with latent variables induces the manifest dynamical system $\left(\mathbb{R}, \mathbb{K}^{q}, \mathcal{B}\right)$ with $\mathcal{B}=(R(d / d t))^{-1}$ im $M(d / d t)$. It can be shown that $\left(\mathbb{R}, \mathbb{K}^{q}, \mathcal{B}\right) \in \mathcal{L}^{q}$, that is, that $(R(d / d t))^{-1}$ im $M(d / d t)$ can itself be described as the solution set of a system of constant coefficient differential equations. In other words, there exists a polynomial matrix $R^{\prime} \in \mathbb{R}^{\bullet \times q}[s]$ such that the manifest behavior of (12) is described by

$$
R^{\prime}\left(\frac{d}{d t}\right) w=0
$$

The matrix $R^{\prime}$ can be obtained as follows. By premultiplying $M$ by a suitable unimodular polynomial matrix $U, U M$ can be brought in the form

$$
U M=\left[\begin{array}{c}
0 \\
\dot{M}^{i \prime}
\end{array}\right]
$$

with $M^{\prime \prime} \in \mathbb{K}_{f r}^{\bullet \times d}[s]$. Partitioning $U R$ conformably as

$$
U R=\left[\begin{array}{c}
R^{\prime} \\
\ddot{R^{\prime \prime}}
\end{array}\right]
$$

yields the desired $R^{\prime}$. The result that (13) then defines the manifest behavior of (12) follows easily from the observation that $M^{\prime \prime}(d / d t)$ is surjective (in other words, if $P \in \mathbb{R}_{f r}^{n_{1} \times n_{2}}[s]$, then $P(d / d t): C^{\infty}\left(\mathbb{R} ; \mathbb{K}^{n_{2}}\right) \rightarrow C^{\infty}\left(\mathbb{R} ; \mathbb{K}^{n_{1}}\right)$ will be a surjective map).

A very useful class of systems with latent variables are the state space systems. In this case, the latent variables are denoted by $x$ instead of by $\ell$. In [15], [16], we have defined state space models abstractly in terms of concatenation of trajectories. For differential systems, the result is that state space systems are precisely those latent variable systems whose full behavior can be described by the following special type of differential equations linking the state trajectory $x \in C^{\infty}\left(\mathbb{R} ; \mathbb{K}^{n}\right)$ to the manifest trajectory $w \in C^{\infty}\left(\mathbb{R} ; \mathbb{K}^{q}\right)$ :

$$
\left(E \frac{d}{d t}+F\right) x+H w=0
$$

with $E, F, H$ matrices over $\mathbb{K}$ of suitable dimension. The crucial feature of (14) is that this differential equation is first-order in $x$ and zeroth-order in $w$.

Let us denote the state space systems with manifest signal space $\mathbb{K}^{q}$, and state space $\mathbb{K}^{n}$ by $\mathcal{L}_{s}^{q, n}$. It follows that $\mathcal{L}_{s}^{q, n}$ is parametrized by $\mathbb{K}^{\bullet \times(2 n+q)}$ by associating with the element $[E: F: H] \in \mathbb{K}^{\bullet \times(2 n+q)}$ the behavioral equations (14). We denote the state space system described by (14) simply as $(E, F, H)$.

It follows immediately from the elimination of latent variables that the manifest behavior of $\Sigma_{s} \in \mathcal{L}_{s}^{q, n}$ leads to a system in $\mathcal{L}^{q}$. However, the converse also holds: for any $\Sigma \in \mathcal{L}^{q}$, i.e., for any polynomial matrix $R \in \mathbb{K}^{\bullet \times q}[s]$, there exist nonnegative integers $n, f \in \mathbb{N}_{0}$ and matrices $E, F \in \mathbb{K}^{f \times n}, H \in \mathbb{K}^{f \times q}$ such that the manifest behavior of (14) is represented by (1). If $\Sigma_{s} \in \mathcal{L}^{q, n}$ induces in this sense the system $\Sigma \in \mathcal{L}^{q}$, then we call $\Sigma_{s}$ (or $\left.(E, F, H) \in \mathbb{K}^{\bullet \times(2 n+q)}\right)$ a state space representation of $\Sigma \in \mathcal{L}^{q}$ ( or of $\left.(1)\right)$. We denote this as $(E, F, H) \sim \Sigma$, or $(E, F, H) \sim R$. 
Let $E, F \in \mathbb{K}^{f \times n}, H \in \mathbb{K}^{f \times q}$, and $(E, F, H) \leadsto \Sigma \in \mathcal{L}^{q}$. We call this state space system minimal if $\left(E^{\prime}, F^{\prime} \in \mathbb{K}^{f^{\prime} \times n}, H^{\prime} \in \mathbb{K}^{f^{\prime} \times q}\right.$, and $\left.\left(E^{\prime}, F^{\prime}, H^{\prime}\right) \sim \Sigma\right)$ implies $\left(f \leq f^{\prime}\right.$ and $\left.n \leq n^{\prime}\right)$. In [15], [16], it is shown that any system $\Sigma \in \mathcal{L}^{q}$ admits a minimal state space representation (in other words, both $f$ and $n$ can be simultaneously minimized). This implies that, in a minimal state representation, the number of state variables will depend on $\Sigma$, but not on the particular minimal state space representation of $\Sigma$. We denote this minimal number of state variables by $n(\Sigma)$. Similarly, $f(\Sigma)$ denotes the minimal number of equations in (14) representing $\Sigma$. It is easy to see [15] that $f(\Sigma)=n(\Sigma)+p(\Sigma)$. The following proposition play a crucial role in our proof of Theorem 3.

Proposition 7. Let $(E, F, H) \leadsto \Sigma \in \mathcal{L}^{q}$ be minimal. Then $\left(E^{\prime}, F^{\prime}, H^{\prime}\right) \leadsto \Sigma$ will also be minimal if and only if there exist $V \in G L\left(\mathbb{K}^{f(\Sigma)}\right)$ and $T \in G L\left(\mathbb{K}^{n(\Sigma)}\right)$ such that

$$
E^{\prime}=V E T, \quad F^{\prime}=V F T, \quad \text { and } \quad H^{\prime}=V H
$$

Finally, for given $(E, F, H)$ and $\left(E^{\prime}, F^{\prime}, H^{\prime}\right)$, this $V$ and $T$ are unique.

Proof. For the proof, see [15].

This proposition states that minimal representations of a given manifest behavior differ only in their choice of the basis in the state space and in the equation space.

THEOREM 8. Let $\rho: G \rightarrow G L\left(\mathbb{K}^{q}\right)$ be a representation of the compact group $G$ on $\mathbb{K}^{q}$. Let $\Sigma \in \mathcal{L}^{q}$ and let $\Sigma_{s} \cong(E, F, H) \in \mathcal{L}^{q, n}$ be a minimal state space representation of $\Sigma$. Then $\Sigma$ is $\rho$-symmetric if and only if there exist representations $\rho^{\prime}: G \rightarrow G L\left(\mathbb{K}^{n(\Sigma)}\right)$ and $\rho^{\prime \prime}: G \rightarrow G L\left(\mathbb{K}^{f(\Sigma)}\right)$ such that

$$
\rho^{\prime \prime} E=E \rho^{\prime}, \quad \rho^{\prime \prime} F=F \rho^{\prime}, \quad \text { and } \quad \rho^{\prime \prime} H=H \rho .
$$

Proof. The "if" part is clear. To see the "only if" part, observe that $\left(E, F, H \rho_{g}\right)$ is also a minimal state space representation of $\Sigma$. By Proposition 7, this implies that there exist $V_{g} \in G L\left(\mathbb{K}^{f(\Sigma)}\right)$ and $T_{g} \in G L\left(\mathbb{K}^{n(\Sigma)}\right)$ such that $E=V_{g} E T_{g}, F=V_{g} F T_{g}$, and $H \rho_{g}=V_{g} H$. Define $\rho^{\prime}: g \mapsto V_{g}$ and $\rho^{\prime \prime}: g \mapsto\left(T_{g}\right)^{-1}$. We claim that $\rho^{\prime}$ and $\rho^{\prime \prime}$ are also representations of $G$. In fact, from the uniqueness condition in Proposition 7 , it follows immediately that $V_{g_{1} g_{2}}=V_{g_{1}} V_{g_{1}}$ and $\left(T_{g_{1} g_{2}}\right)^{-1}=\left(T_{g_{1}}\right)^{-1}\left(T_{g_{2}}\right)^{-1}$.

9. Canonical forms for state space models of symmetric systems. We now use Theorem 8 and the ideas of $\S 6$ to obtain canonical forms for state space systems. It is convenient to distinguish again between the complex case where $\mathbb{K}=\mathbb{C}$ and the real case where $\mathbb{K}=\mathbb{R}$.

9.1. Complex state space systems. Write the representations $\rho, \rho^{\prime}$, and $\rho^{\prime \prime}$ of Theorem 8 in terms of irreducible representations as

$$
\begin{aligned}
& \rho \cong m_{1} \rho_{1} \oplus m_{2} \rho_{2} \oplus \cdots \oplus m_{k} \rho_{k} \\
& \rho^{\prime} \cong m_{1}^{\prime} \rho_{1} \oplus m_{2}^{\prime} \rho_{2} \oplus \cdots \oplus m_{k}^{\prime} \rho_{k} \\
& \rho^{\prime \prime} \cong m_{1}^{\prime \prime} \rho_{1} \oplus m_{2}^{\prime \prime} \rho_{2} \oplus \cdots \oplus m_{k}^{\prime \prime} \rho_{k}
\end{aligned}
$$

Now choose a $\rho$-adapted basis in the manifest signal space $\mathbb{C}^{q}$, a $\rho^{\prime}$-adapted basis in the state space $\mathbb{C}^{n(\Sigma)}$, and a $\rho^{\prime \prime}$-adapted basis in the equation space $\mathbb{C}^{f(\Sigma)}$. Now apply Schur's lemma to (15). This yields that, in these bases $E, F$, and $H$ will take the 
form

$$
\begin{gathered}
E=\operatorname{diag}\left(E_{1} \otimes I_{n_{1}}, E_{2} \otimes I_{n_{2}}, \ldots, E_{k} \otimes I_{n_{k}}\right), \\
F=\operatorname{diag}\left(F_{1} \otimes I_{n_{1}}, F_{2} \otimes I_{n_{2}}, \ldots, F_{k} \otimes I_{n_{k}}\right), \\
H=\operatorname{diag}\left(H_{1} \otimes I_{n_{1}}, H_{2} \otimes I_{n_{2}}, \ldots, H_{k} \otimes I_{n_{k}}\right) .
\end{gathered}
$$

This yields the following result.

THEOREM 9. Let $G$ be a compact group and let $\rho: G L\left(\mathbb{C}^{q}\right)$ be a representation of $G$ on $\mathbb{C}^{q}$. Assume that $\rho \cong m_{1} \rho_{1} \oplus m_{2} \rho_{2} \oplus \cdots \oplus m_{k} \rho_{k}$ with $\rho_{i}: G \rightarrow G L\left(\mathbb{C}^{n_{i}}\right)$, $i=1,2, \ldots, k$, distinct irreducible representations. Assume that the basis in $\mathbb{C}^{q}$ is $\rho$-adapted, as in Theorem 4. Then $\Sigma=\left(\mathbb{R}, \mathbb{C}^{q}, \mathcal{B}\right) \in \mathcal{L}^{q}$ is $\rho$-symmetric if and only if there exist $m_{i}^{\prime}, m_{i}^{\prime \prime} \in \mathbb{Z}_{+}$and matrices $E_{i}, F_{i} \in \mathbb{C}^{m_{i}^{\prime \prime} \times m_{i}^{\prime}}, H_{i} \in \mathbb{C}^{m_{i}^{\prime \prime} \times m_{i}}$ such that $\Sigma$ admits the minimal state space representation of the form

$$
\left(\left(E_{i} \frac{d}{d t}+F_{i}\right) \otimes I_{n_{i}}\right) x_{i}+\left(H_{i} \otimes I_{n_{i}}\right) \tilde{w}_{i}=0, \quad i=1,2, \ldots, k
$$

with $\tilde{w}$ as in Theorem 4 , and where $x_{i}: \mathbb{R} \rightarrow\left(\mathbb{C}^{n_{i}}\right)^{m_{i}^{\prime}}, x=\operatorname{col}\left(x_{1}, x_{2}, \ldots, x_{k}\right)$, is the state trajectory.

9.2. Real state space systems. To obtain a canonical form for real symmetric state space systems, we proceed in complete analogy to $\S 6.2$. By considering the components of real, complex, and quaternionic type in the decomposition of $\rho$, the following result is obtained.

THEOREM 10. Let $G$ be a compact group and let $\rho: G \rightarrow G L\left(\mathbb{R}^{q}\right)$ be a representation of $G$ on $\mathbb{R}^{q}$. Assume that $\rho$ is decomposed as in (8) and that the basis in $\mathbb{R}^{q}$ is $\rho$-adapted, as in Theorem 5 . Then $\Sigma=\left(\mathbb{R}, \mathbb{R}^{q}, \mathcal{B}\right) \in \mathcal{L}^{q}$ is $\rho$-symmetric if and only if there exist

$$
m_{\mathbb{R}, i}^{\prime}, m_{\mathbb{C}, i}^{\prime}, m_{\mathbb{H}, i}^{\prime}, m_{\mathbb{R}, i}^{\prime \prime}, m_{\mathbb{C}, i}^{\prime \prime}, m_{\mathbb{H}, i}^{\prime \prime} \in \mathbb{Z}_{+}
$$

and matrices

$$
\begin{aligned}
& E_{\mathbb{R}, i}, F_{\mathbb{R}, i} \in \mathbb{R}^{m_{\mathbb{R}, i}^{\prime \prime} \times m_{\mathbb{R}, i}^{\prime}}, H_{\mathbb{R}, i} \in \mathbb{R}^{m_{\mathbb{R}, i}^{\prime \prime} \times m_{\mathbb{R}, i}} ; \\
& E_{\mathbb{C}, i}, F_{\mathbb{C}, i} \in \mathbb{C}^{m_{\mathbb{C}, i}^{\prime \prime} \times m_{\mathbb{C}, i}^{\prime}}, H_{\mathbb{C}, i} \in \mathbb{C}^{m_{\mathbb{C}, i}^{\prime \prime} \times m_{\mathbb{C}, i}} ; \\
& E_{\mathbb{H}, i}, F_{\mathbb{H}, i} \in \mathbb{H}^{m_{\mathbb{H}, i}^{\prime \prime} \times m_{\mathbb{H}, i}^{\prime}}, H_{\mathbb{H}, i} \in \mathbb{C}^{m_{\mathbb{H}, i}^{\prime \prime} \times m_{\mathbb{H}, i}},
\end{aligned}
$$

such that $\Sigma$ admits a minimal state space representation of the form

$$
\begin{aligned}
& \left(\left(E_{\mathbb{R}, i} \frac{d}{d t}+F_{\mathbb{R}, i}\right) \otimes I_{n_{\mathbb{R}, i}}\right) x_{\mathbb{R}, i}+\left(H_{\mathbb{R}, i} \otimes I_{n_{\mathbb{R}, i}}\right) \tilde{w}_{\mathbb{R}, i}=0, \quad i=1,2, \ldots, k_{\mathbb{R}}, \\
& (17)\left(\left(E_{\mathbb{C}, i} \frac{d}{d t}+F_{\mathbb{C}, i}\right) \otimes I_{n_{\mathbb{C}, i}}\right) x_{\mathbb{C}, i}+\left(H_{\mathbb{C}, i} \otimes I_{n_{\mathbb{C}, i}}\right) \tilde{w}_{\mathbb{C}, i}=0, \quad i=1,2, \ldots, k_{\mathbb{C}}, \\
& \left(\left(E_{\mathbb{H}, i} \frac{d}{d t}+F_{\mathbb{H}, i}\right) \otimes I_{n_{\mathbb{H}, i}}\right) x_{\mathbb{H}, i}+\left(H_{\mathbb{H}, i} \otimes I_{n_{\mathbb{H}, i}}\right) \tilde{w}_{\mathbb{H}, i}=0, \quad i=1,2, \ldots, k_{\mathbb{H}}
\end{aligned}
$$

with $\tilde{w}$ as in Theorem 5 , and where $x=\operatorname{col}\left(x_{\mathbb{R}}, x_{\mathbb{C}}, x_{\mathbb{H}}\right)$ is the state trajectory;

$$
\begin{array}{ll}
x_{\mathbb{R}}=\operatorname{col}\left(x_{\mathbb{R}, 1}, x_{\mathbb{R}, 2}, \ldots, x_{\mathbb{R}, k_{\mathbb{R}}}\right), & \text { with } x_{\mathbb{R}, i}: \mathbb{R} \rightarrow\left(\mathbb{R}^{n_{\mathbb{R}, i}}\right)^{m_{\mathbb{R}, i}^{\prime}} \\
x_{\mathbb{C}}=\operatorname{col}\left(x_{\mathbb{C}, 1}, x_{\mathbb{C}, 2}, \ldots, x_{\mathbb{C}, k_{\mathbb{C}}}\right), & \text { with } x_{\mathbb{C}, i}: \mathbb{R} \rightarrow\left(\mathbb{C}^{n_{\mathbb{C}, i}}\right)^{m_{\mathbb{C}, i}^{\prime}} \\
x_{\mathbb{H}}=\operatorname{col}\left(x_{\mathbb{H}, 1}, x_{\mathbb{H}, 2}, \ldots, x_{\mathbb{H}, k_{\mathbb{H}}}\right), & \text { with } x_{\mathbb{H}, i}: \mathbb{R} \rightarrow\left(\mathbb{H}^{n_{\mathbb{H}, i}}\right)^{m_{\mathbb{H}, i}^{\prime}}
\end{array}
$$




\section{Proof of Theorem 3.}

10.1. The complex case. As already shown in $\S 6$, it suffices to prove that, if $\Sigma=\left(\mathbb{R}, \mathbb{C}^{q}, \mathcal{B}\right) \in \mathcal{L}^{q}$ is $\rho$-symmetric, then it allows a minimal representation, as given in Theorem 4. By Theorem 9, it allows a minimal state space representation as (16). Now consider in the $i$ th equation of (16), $x_{i}$ as a latent variable. Using the elimination of latent variables procedure as explained in the beginning of $\S 8$, we can conclude that the $i$ th equation of (16) constrains $\tilde{w}_{i}$ to satisfy an equation of the form $\left(\Lambda_{i}(d / d t) \otimes I_{n_{i}}\right) \tilde{w}_{i}=0$. This yields the $i$ th representation of $(7)$ and results in the desired representation of Theorem 4 .

10.2. The real case. For the real case, we can use this elimination of latent variables procedure unchanged for each of the real and complex equations in (17). These will yield the corresponding real, respectively complex, term in (11) of Theorem 5. However, the quaternionic equations in (17) require separate attention, since the quaternions $\mathbb{H}$ do not form a field. This brings us to the following algebraic excursion.

Let $\mathcal{R}$ be a ring with an identity. Let $\mathcal{R}[s]$ denote the ring of polynomials with coefficients in $\mathcal{R}$, and $\mathcal{R}^{n_{1} \times n_{2}}[s]$ the $n_{1} \times n_{2}$ matrices with elements in $\mathcal{R}[s]$. Furthermore, let $G L(n, \mathcal{R}[s])$ denote the group of units of the ring $\mathcal{R}^{n \times n}[s]$, i.e., the set of polynomial matrices over $\mathcal{R}$ with a polynomial inverse. We call these matrices unimodular. Now consider the problem of bringing a given element $P \in \mathcal{R}^{n_{1} \times n_{2}}[s]$ into a convenient canonical form by premultiplying it by a suitable element $U_{1} \in G L\left(n_{1}, \mathcal{R}[s]\right)$ and postmultiplying it by a suitable element $U_{2} \in G L\left(n_{2}, \mathcal{R}[s]\right)$. We will say that $P$ can be brought in diagonal form if there exist such $U_{1}, U_{2}$ yielding for $U_{1} P U_{2}$ a matrix of the form

$$
U_{1} P U_{2}=\left[\begin{array}{ccc}
D & \vdots & 0 \\
\cdots & \vdots & \cdots \\
0 & \vdots & 0
\end{array}\right]
$$

with $D$ a diagonal polynomial matrix. It is well known that any $P$ can be brought in diagonal form if $\mathcal{R}=\mathbb{R}$ or $\mathbb{C}$.

For what rings $\mathcal{R}$ such a diagonalization is possible? We now show that it is sufficient for $\mathcal{R}$ to be a division ring. Recall that a ring is a division ring (also called a skew field) if its nonzero elements form a group $(\mathcal{R}$ must contain an identity distinct from the zero, and $a \neq 0$ must imply that it is a unit of $\mathcal{R}$ (i.e., it has an inverse $\left.\left.a^{-1} \in \mathcal{R}\right)\right)$.

Lemma 11. Let $\mathcal{R}$ be a division ring. Then each $P \in \mathcal{R}^{n_{1} \times n_{2}}[s]$ can be brought in diagonal form.

Proof. The proof is an adaption of the case of a skew field of the proof of the Smith form for real or complex polynomial matrices as given in [3].

If $P=0$, there is nothing to prove. Otherwise, let $P_{k \ell}$ be the element of $P$ of least degree. By permuting rows and columns we can assume that this element is in $(1,1)$ entry. Now assume that the $(1,2)$ entry is also nonzero. Then divide $P_{12}$ by $P_{11}$ with remainder, yielding $P_{12}=P_{11} d+r$ with degree $r<$ degree $P_{11}$. Now postmultiply with the unimodular matrix

$$
\left[\begin{array}{ccccc}
1 & -d & 0 & \cdots & 0 \\
0 & 1 & 0 & \cdots & 0 \\
\vdots & \vdots & \vdots & \ddots & \vdots \\
0 & 0 & 0 & \cdots & 1
\end{array}\right]
$$


This replaces the element $P_{12}$ by $r$. If $r \neq 0$, move it to the $(1,1)$ position. Repeat this process for each element of the first row and first column (using the division with remainder $P_{21}=d P_{11}+r$ and premultiplication). Each time the division is carried out with a nonzero remainder, the degree of the $(1,1)$ element must decrease. Hence after a finite number of steps, we will obtain a matrix of the form

$$
\left[\begin{array}{ccccc}
* & 0 & 0 & \cdots & 0 \\
0 & * & * & \cdots & * \\
\vdots & \vdots & \vdots & \ddots & \vdots \\
0 & * & * & \cdots & *
\end{array}\right]
$$

and we obtain the lemma by induction.

Now consider the ring $\mathbb{H}$ of quaternions over $\mathbb{R}$ : expressions of the type $\alpha+i \beta+$ $j \gamma+k \delta$ with $\alpha, \beta, \gamma, \delta \in \mathbb{R}$ and $i, j, k$ elements satisfying the multiplication rules as in Example 8. With the obvious rules of addition and multiplication, $\mathbb{H}$ is a ring. However, $\mathbb{H}$ is not commutative, but it is easy to see that it is a division ring. Hence a polynomial matrix $P \in \mathbb{H}^{n_{1} \times n_{2}}[s]$ can be brought in diagonal form.

Now consider a system of differential equations.

$$
R\left(\frac{d}{d t}\right) w=M\left(\frac{d}{d t}\right) \ell
$$

where $w \in C^{\infty}\left(\mathbb{R} ; \mathbb{H}^{q}\right), \ell \in C^{\infty}\left(\mathbb{R} ; \mathbb{H}^{d}\right), R \in \mathbb{H}^{f \times q}[s]$, and $M \in \mathbb{H}^{f \times d}[s]$. Note that this is a differential equation with latent variables as (12) but in which the signals and coefficients take their values in the skew field of quaternions $\mathbb{H}$. We would like to eliminate the latent variables in (19), using only operations in $\mathbb{H}$.

Observe that the manifest behavior of (19) remains invariant if we replace $R$ with $U_{1} R$ and $M$ with $U_{1} M U_{2}$ with $U_{1}$ and $U_{2}$ both unimodular polynomial matrices with coefficients in $\mathbb{H}$. By Lemma $11, U_{1}$ and $U_{2}$ can be chosen such that

$$
U_{1} M U_{2}=\left[\begin{array}{c}
0 \\
\ddot{D}
\end{array}\right]
$$

with $D=\operatorname{diag}\left(d_{1}, d_{2}, \ldots, d_{r}\right)$ and $0 \neq d_{i} \in \mathbb{H}[s]$. Denote the conformable partition of $U_{1} R$ by

$$
U_{1} R=\left[\begin{array}{l}
R^{\prime} \\
\ddot{R}^{\prime \prime}
\end{array}\right]
$$

Now observe that, if $0 \neq d \in \mathbb{H}[s]$, then the operator $d(d / d t): C^{\infty}(\mathbb{R} ; \mathbb{H}) \rightarrow C^{\infty}(\mathbb{R} ; \mathbb{H})$ is surjective. To see this, it suffices to write this as a differential operator from $C^{\infty}\left(\mathbb{R} ; \mathbb{R}^{4}\right)$ into itself and use the fact that the corresponding $(4 \times 4)$ polynomial matrix with real coefficients has a nonzero determinant.

This implies that the manifest behavior of (19) is governed by

$$
R^{\prime}\left(\frac{d}{d t}\right) w=0
$$

Applying this elimination result to each of the quaternionic equations in (17) yields the corresponding quaternionic equations in (11).

This establishes that Theorem 10 yields the desired representation of Theorem 5 . 
11. Group representations in $G L(n, \mathbb{K}[s])$. Theorem 3 implies an interesting result about abstract group representations. A mapping $\rho: G \rightarrow G L(n, \mathbb{K}[s])$ (the unimodular $n \times n$ polynomial matrices over $\mathbb{K})$, which is a group homomorphism and continuous (in the sense that the map $g \in G \mapsto \rho_{g}(\lambda) \in G L\left(\mathbb{K}^{n}\right)$ is continuous for each fixed $\lambda \in \mathbb{K}$ and the map $\lambda \in \mathbb{K} \mapsto \rho_{g}(\lambda) \in G L\left(\mathbb{K}^{n}\right)$, is continuous uniformly in $g \in G)$, is called a representation of $G$ on $G L(n, \mathbb{K}[s])$. Two such representations $\rho_{1}$ and $\rho_{2}$ are said to be isomorphic if there exist a $U \in G L(n, \mathbb{K}[s])$ such that $\rho_{2, g}(s)=$ $U(s) \rho_{1, g}(s)(U(s))^{-1}$. The representation $\rho$ is said to be a constant representation if $\rho_{g}(s)$ is actually a constant matrix for all $g \in G$, that is, if $\rho: G \rightarrow G L\left(\mathbb{K}^{q}\right)$, if $\rho$ is actually a representation of $G$ on $\mathbb{K}^{n}$. We now show that Theorem 3 implies that every representation of $G$ on $G L(n, \mathbb{K}[s])$ is isomorphic to a constant one. This result can be deduced from [7], where a more general theorem is proved using very different methods. Our proof, which, as we have seen, relies on the state space representation of dynamical systems in $\mathcal{L}^{q}$, provides a nice alternative "system theoretic" proof of this result in the theory of algebraic groups. On the other hand, we also note that Theorem 3 can be easily deduced directly from Corollary 12 (and hence from [7]). Hence Theorem 3 and Corollary 12 are fully equivalent.

COROLlaRY 12. Let $G L(n, \mathbb{K}[s])$ denote the group of unimodular $n \times n$ polynomial matrices over $\mathbb{K}(=\mathbb{R}$ or $\mathbb{C})$. Let $G$ be a compact group and let $\rho: G \rightarrow G L(n, \mathbb{K}[s])$ be a representation of $G$ on $G L(n, \mathbb{K}[s])$. Then $\rho$ is isomorphic to a constant representation.

Proof. 1) Consider, for each $\lambda \in \mathbb{K}$, the mapping $\rho^{\lambda}: G \rightarrow G L\left(\mathbb{K}^{n}\right)$ defined by $\rho_{g}^{\lambda}:=\rho_{g}(\lambda)$. It is easy to see that $\rho^{\lambda}$ is a representation of $G$ on $\mathbb{K}^{n}$. We first prove that all the representations $\rho^{\lambda}$ are isomorphic. It is well known that two representations of $G$ on $\mathbb{K}^{n}$ are isomorphic if and only if their characters are equal. Hence it suffices to prove that the characters $X_{\rho^{\lambda}}: G \rightarrow \mathbb{K}$, defined by $X_{\rho^{\lambda}}(g):=\operatorname{Trace}\left(\rho_{g}(\lambda)\right)$, are independent of $\lambda \in \mathbb{K}$. Let $\rho^{\lambda} \cong m_{1}^{\lambda} \rho_{1} \oplus m_{2}^{\lambda} \rho_{2} \oplus \cdots \oplus m_{k}^{\lambda} \rho_{k} \oplus \cdots$ be a decomposition of $\rho^{\lambda}$ in terms of irreducible representations. We prove that $m_{k}^{\lambda}$ is independent of $\lambda$. Now $m_{k}^{\lambda}$ is given by $m_{k}^{\lambda}=<\chi_{\rho^{\lambda}}, \chi_{\rho_{k}}:=\int_{G} \chi_{\rho^{\lambda}} \chi_{\rho_{k}}^{*} d g$, where $d g$ is the normalized Haar measure [9] of $G$. This implies that $m_{k}^{\lambda}$ is continuous as a function of $\lambda$ for $\lambda \in \mathbb{K}$. However, since $m_{k}^{\lambda}$ it is integer-valued, $m_{k}^{\lambda}$ must therefore be constant for $\lambda \in \mathbb{K}$. This shows that all the $\rho^{\lambda}$ 's are isomorphic.

2) The first part of the proof yields the existence of a map $M: \mathbb{K} \rightarrow G L\left(\mathbb{K}^{n}\right)$ such that

$$
\rho_{g}(\lambda) M(\lambda)=M(\lambda) \rho_{g}(0)
$$

for all $\lambda \in \mathbb{K}$ and $g \in G$. We now show that this implies the existence of a polynomial matrix $R \in \mathbb{K}^{n \times n}[s]$ with $\operatorname{det} R \neq 0$ such that

$$
\rho_{g}(s) R(s)=R(s) \rho_{g}(0)
$$

for all $g \in G$. In other words, we show that the set of equations

$$
\rho_{g}(s) X(s)=X(s) \rho_{g}(0), \quad g \in G
$$

has a polynomial solution $X \in \mathbb{K}^{n \times n}[s]$ with $\operatorname{det} X \neq 0$. Note that we can rewrite (22) in vector-matrix notation as

$$
A_{g}(s) x(s)=0, \quad g \in G
$$


with $x \in \mathbb{K}^{n^{2}}[s]$ and $A_{g} \in \mathbb{K}^{n^{2} \times n^{2}}[s]$. The vector $x$ corresponds to the elements of $X$ in (22), while the matrix $A_{g}$ corresponds to the coefficients of the linear equations in (22). Since (23) must be satisfied for each $g \in G$, there are, in principle, an infinite number of linear equations in (23). Now consider the rows of the matrices $A_{g}$ and view them as vectors of rational functions, as elements of $\mathbb{K}^{1 \times n^{2}}(s)$. Write (23) as

$$
\left[\begin{array}{c}
A^{\prime}(s) \\
\cdots \\
A^{\prime \prime}(s)
\end{array}\right] x(s)=0
$$

with $A^{\prime}$ such that its rows form a basis over $\mathbb{K}(\sim)$ for the span of the rows of all the matrices $A_{g}, g \in G$. Now observe that, by premultiplying $x$ and $A^{\prime}$ by a unimodular matrix, we may as well assume that $A^{\prime}$ is in Smith form, as follows:

$$
A^{\prime}(s)=[D(s) \vdots 0]
$$

with $D(s)=\operatorname{diag}\left(d_{1}(s), d_{2}(s), \ldots, d_{k}(s)\right)$ and $d_{i} \neq 0$ for $i=1,2, \ldots, k$. This yields that in a conformable partition $A^{\prime \prime}$ will be of the form $A^{\prime \prime}(s)=\left[\tilde{A}^{\prime \prime}(s) \vdots 0\right]$. Obviously, this implies that each vector polynomial

$$
x(s)=\operatorname{col}\left(x_{1}(s), \ldots, x_{k}(s), x_{k+1}(s), \ldots, x_{n^{2}}(s)\right)
$$

with $x_{1}=x_{2}=\cdots=x_{k}=0$ yields a solution $X(s)$ of $(23)$.

To show that there is at least one of these solutions with det $X \neq 0$, we will use (20). This equation yields, for each $\lambda \in \mathbb{K}$, a solution $m(\lambda)$ to (23) with, in (23), $s$ replaced by $\lambda$. Pick a $\tilde{\lambda} \in \mathbb{K}$ such that $d_{i}(\tilde{\lambda}) \neq 0$ for $i=1,2, \ldots, k$. Clearly, $m(\tilde{\lambda})$ must be of the form $\operatorname{col}\left(0, \ldots, 0, \tilde{m}_{k+1}, \ldots, \tilde{m}_{n^{2}}\right)$. Now pick a solution $x(s)$ of $(23)$ such that $x(\tilde{\lambda})=m(\tilde{\lambda})$. The solution $X(s)$ of $(22)$ thus obtained will have $X(\tilde{\lambda})=M(\tilde{\lambda})$, whence $\operatorname{det} X \neq 0$. This shows that (21) has a solution with $\operatorname{det} R \neq 0$.

3) Now consider the dynamical system $\Sigma=\left(\mathbb{R}, \mathbb{K}^{n}, \operatorname{ker} R(d / d t)\right)$. Obviously, by (21), $\Sigma$ is $\rho(0)$-symmetric, and $R(d / d t) w=0$ is a minimal representation of $\Sigma$. By Theorem 3 , there exists a $U \in G L(n, \mathbb{K}[s])$ and a representation $\rho^{\prime}: G \rightarrow G L\left(\mathbb{K}^{n}\right)$ such that

$$
\rho_{g}^{\prime} U(s) R(s)=U(s) R(s) \rho_{g}(0)
$$

for all $g \in G$. Comparing (21) and (24) yields

$$
\rho_{g}(s)=(U(s))^{-1} \rho_{g}^{\prime} U(s)
$$

which is the claim of the corollary.

Remark. Let $\rho: G \rightarrow G L(q, \mathbb{K}[s])$ be a representation of $G$ on $G L(q, \mathbb{K}[s])$. Let $\Sigma=\left(\mathbb{R}, \mathbb{K}^{q}, \mathcal{B}\right) \in \mathcal{L}^{q}$ be $\rho$-symmetric, meaning that $\rho_{g}(d / d t) \mathcal{B}=\mathcal{B}$ for all $g \in G$. Note that this is a dynamic symmetry, in contrast (see §3.3) with the static symmetries studied in this paper. However, Corollary 12 shows that, by a dynamic change of variables $w \mapsto U(d / d t) w$, with $U$ a suitable unimodular polynomial matrix, the study of this type of dynamic symmetry reduces to a static symmetry. 


\section{REFERENCES}

[1] R. W. BRockett AND J. L. Willems, Discreted partial differential equations: Examples of control systems defined on modules, Automatica, 10 (1974), pp. 507-515.

[2] F. Fagnani and J. C. Willems, Representations of time-reversible systems, J. Math. Systems Estimation and Control, 1 (1991), pp. 5-28.

[3] P. A. Furhmann, Linear Systems and Operators in Hilbert Space, McGraw-Hill, New York, 1981.

[4] J. W. GRIZzLE AND S. I. MARCUS, The structure of nonlinear control systems possessing symmetries, IEEE Trans. Automat. Control, 30 (1985), pp. 248-258.

[5] M. HAzEWINKel AND C. F. MARTIN, Symmetric linear systems: An application of algebraic systems theory, Internat. J. Control, 37 (1983), pp. 1371-1384.

[6] - On decentralization, symmetry, and special structure in linear systems, Lecture Notes in Control and Inform. Sci., 58 (1984), pp. 437-440.

[7] V. G. KAC AND D. H. Peterson, On geometric invariant theory for infinite-dimensional groups, Lecture Notes in Math., 1271 (1987), pp. 109-142.

[8] C. F. MARTIN, Linear decentralized systems with special structure, Internat. J. Control, 35 (1982), pp. 291-308.

[9] J.-P. SERrE, Linear Representations of Finite Groups, Springer-Verlag, 1977, Berlin, New York, translation of Représentations Linéaires des Groupes Finis, Hermann, 1971.

[10] J. M. Schumacher, Transformations of linear systems under external equivalence, J. Linear Algebra Its Appl., 102 (1988), pp. 1-34.

[11] A. J. VAN DER SCHAFT, Symmetries, conversation laws, and time reversibility for Hamiltonian systems with external forces, J. Math. Phys., 24 (1983), pp. 2095-2101.

[12] - System Theoretic Descriptions of Physical Systems, CWI Tract No. 3, 1984.

[13] - Symmetries in optimal control, SIAM J. Control and Optim., 25 (1987), pp. 245-259.

[14] J. C. W Illems, Symmetries in dynamical systems, in Proc. 25th IEEE Conference on Decision and Control, Athens, Greece, 1986, pp. 520-521.

[15] — Paradigms and puzzles in the theory of dynamical systems, IEEE Trans. on Automat. Control, 36 (1991), pp. 259-294.

[16] — Models for dynamics, Dynam. Report. Ser. Dynam. Syst. Appl., 2 (1989), pp. 171-269.

[17] D. C. Youla AND P. TISsI, N-port synthesis via reactance extraction, IEEE Internat. Convention Record, 1966, pp. 183-205. 